\title{
METANILPOTENT VARIETIES OF GROUPS
}

\author{
R. M. BRYANT and A. N. KRASIL'NIKOV
}

(Received 10 February 2000)

Communicated by R. B. Howlett

\begin{abstract}
For each positive integer $n$ let $\mathbf{N}_{2, n}$ denote the variety of all groups which are nilpotent of class at most 2 and which have exponent dividing $n$. For positive integers $m$ and $n$, let $\mathbf{N}_{2, m} \mathbf{N}_{2, n}$ denote the variety of all groups which have a normal subgroup in $\mathbf{N}_{2, m}$ with factor group in $\mathbf{N}_{2, n}$. It is shown that if $G \in \mathbf{N}_{2, m} \mathbf{N}_{2, n}$, where $m$ and $n$ are coprime, then $G$ has a finite basis for its identities.
\end{abstract}

2000 Mathematics subject classification: primary $20 \mathrm{E} 10$.

\section{Introduction}

The finite basis question for a group $G$ asks whether the set of all identities of $G$ is equivalent to some finite set of identities. (We refer to [13] for terminology and basic results concerned with varieties of groups, but we use the term 'identity' rather than 'law'.) Between 1970 and 1973 a number of examples were published of groups for which the answer is negative: see [9] for references covering this period and see [5] for an account of more recent results. In the majority of these examples, the groups are metanilpotent (that is, nilpotent-by-nilpotent) and have finite exponent. In the simplest cases the groups belong to the variety $\mathbf{N}_{2,4} \mathbf{N}_{2,4}$ : here, for any positive integer $n, \mathbf{N}_{2, n}$ denotes the variety of all groups which are nilpotent of class at most 2 and have finite exponent dividing $n$, and, for varieties $\mathbf{U}$ and $\mathbf{V}, \mathbf{V U}$ denotes the product variety, consisting of all groups which have a normal subgroup in $\mathbf{V}$ with factor group in $\mathbf{U}$. However, there are also many positive results. In particular, Lyndon [11] showed that every nilpotent group has a finite basis for its identities and Krasil'nikov [10] showed, much more generally, that the same is true for every nilpotent-by-abelian group.

Work supported by EPSRC grant GR/L21273.

(C) 2002 Australian Mathematical Society $1446-7887 / 2000 \$ A 2.00+0.00$ 
In the negative examples mentioned above in which $G$ is metanilpotent of finite exponent there is no bound on the class of the nilpotent subgroups of $G$. It seems still to be an open question whether a soluble group of finite exponent, in which the nilpotent subgroups have bounded class, has a finite basis for its identities. Our main result gives a positive answer in many simple cases.

THEOREM A. Let $G \in \mathbf{N}_{2, m} \mathbf{N}_{2, n}$ where $m$ and $n$ are coprime positive integers. Then $G$ has a finite basis for its identities.

A special case of this result was proved by Brady, Bryce and Cossey [2]: they showed that $G$ has a finite basis for its identities if $G$ belongs to $\mathbf{A}_{m} \mathbf{N}_{2, n}$, where $m$ and $n$ are coprime positive integers and $\mathbf{A}_{\boldsymbol{m}}$ denotes the variety of all abelian groups of exponent dividing $m$. Theorem A solves a problem posed by Kovács and Newman [9]. The method adopted in [2] depends upon an analysis of the irreducible linear groups in $\mathbf{N}_{2, n}$, in prime characteristic not dividing $n$, and develops ideas of Higman [8]. However, at about the same time, Cohen [4] introduced a quite different method for tackling the finite basis question, dependent on the combinatorics of ordered sets. Cohen used this method to prove that every metabelian group has a finite basis for its identities, and the method was developed by others in later work such as $[3,10]$ and [12]. We apply similar methods here, for which we need the idea of a well-quasiordered set, defined as follows.

A quasi-order on a set $W$ is a binary relation $\preccurlyeq$ on $W$ which is reflexive and transitive. (We do not assume that $x \preccurlyeq y$ and $y \preccurlyeq x$ imply $x=y$, as in a partial order. Furthermore, we give no meaning to $\prec$, only to $\preccurlyeq$.) As shown in [6], the following two properties of a quasi-ordered set $(W, \preccurlyeq)$ are equivalent:

(i) for every infinite sequence $w_{1}, w_{2}, \ldots$ of elements of $W$ there exist $i$ and $j$ with $i<j$ such that $w_{i} \preccurlyeq w_{j}$;

(ii) for every subset $X$ of $W$ there exists a finite subset $Y$ of $X$ such that for every element $x$ of $X$ there exists $y \in Y$ such that $y \preccurlyeq x$.

If (either of) these conditions hold then $(W, \preccurlyeq)$ is said to be well-quasi-ordered. If the relation $\preccurlyeq$ is a total (or linear) order then we obtain the more familiar idea of a well-ordered set.

We need to apply this idea to bilinear forms. Let $K$ be a non-zero, finite, commutative and associative ring, with identity element, and let $S$ be a finitely generated $K$-module. By an $S$-form we mean a pair $(V, \theta)$ consisting of a finitely generated, non-zero, free $K$-module $V$ and a $K$-bilinear mapping $\theta: V \times V \rightarrow S$. If $(V, \theta)$ and $\left(V^{\prime}, \theta^{\prime}\right)$ are $S$-forms we write $(V, \theta) \preccurlyeq\left(V^{\prime}, \theta^{\prime}\right)$ if there is a $K$-module monomorphism $\xi: V \rightarrow V^{\prime}$ such that $\theta\left(v_{1}, v_{2}\right)=\theta^{\prime}\left(v_{1} \xi, v_{2} \xi\right)$ for all $v_{1}, v_{2} \in V$. The first step in the proof of Theorem $A$ is the following result (or, to be precise, a more technical version of this result stated in Section 3). 
THEOREM B. The set of all $S$-forms is well-quasi-ordered under the relation $\preccurlyeq$.

Strictly speaking, the class of all $S$-forms is not a set. However, Theorem B can be rephrased to say that every set of $S$-forms is well-quasi-ordered under $\preccurlyeq$.

A result like this for trilinear alternating forms over a finite field was obtained by Atkinson [1] in order to prove a different finite basis result.

The finite basis question for a group $G$ is equivalent to the finite basis question for the variety $\mathbf{V}$ generated by $G$ (see [13]). Furthermore, if $F$ is a free group of countably infinite rank and $\mathbf{V}(F)$ denotes the verbal subgroup of $F$ corresponding to $\mathbf{V}$ then every subvariety of $\mathbf{V}$ is finitely based if and only if $\mathbf{V}$ is finitely based and the maximal condition holds for fully invariant subgroups of the relatively free group $F / \mathrm{V}(F)$. Much of the proof of Theorem A is concerned with establishing that the maximal condition holds in some closely related situations, typically for certain ideals in group algebras.

Let $n$ be a positive integer and let $A$ be a free group of countably infinite rank in the variety $\mathbf{N}_{2, n}$. Let $\mathbb{F}$ be a field of characteristic which does not divide $n$. Let $\Psi$ be the set of all endomorphisms of $A$ and, for each positive integer $r$, let $A^{\times r}$ denote the $r$-th direct power of $A$. Each element $\psi$ of $\Psi$ acts 'diagonally' on $A^{\times r}$ by $\left(a_{1}, \ldots, a_{r}\right) \psi=\left(a_{1} \psi, \ldots, a_{r} \psi\right)$ for all $a_{1}, \ldots, a_{r} \in A$, and this action can be extended to the group algebra $\mathbb{F}\left(A^{\times r}\right)$ in the obvious way. Using the version of Theorem $B$ mentioned above we shall prove the following result.

THEOREM C. For each positive integer $r$, the maximal condition holds for $\Psi$-closed left ideals of $\mathbb{F}\left(A^{\times r}\right)$.

If $U$ is a left $C$-module, for some algebra $C$, and if there is also an action of $\Psi$ on $U$, we call $U$ a $(C, \Psi)$-module. The concepts of $(C, \Psi)$-submodule and homomorphism of $(C, \Psi)$-modules are defined in the obvious way.

The algebra $\mathbb{F}(A \times A)$ is isomorphic to $\mathbb{F} A \otimes \mathbb{F} A$ (where the tensor product is taken over $\mathbb{F}$ ) under the linear map which sends $\left(a, a^{\prime}\right)$ to $a \otimes a^{\prime}$ for all $a, a^{\prime} \in A$. We shall identify these two algebras and write $\left(a, a^{\prime}\right)$ or $a \otimes a^{\prime}$ interchangeably. Let $R$ be the subspace of $\mathbb{F}(A \times A)$ spanned by all elements of the form $a \otimes a$ and $a \otimes a^{\prime}+a^{\prime} \otimes a$ for $a, a^{\prime} \in A$. It is easily verified that $R$ is a subalgebra of $\mathbb{F}(A \times A)$. Thus we may regard $\mathbb{F}(A \times A)$ as a left $R$-module and, indeed, as an $(R, \Psi)$-module. Clearly $R$ is an $(R, \Psi)$-submodule of $\mathbb{F}(A \times A)$. The last main step in the proof of Theorem $\mathrm{A}$ is the following result.

THEOREM D. The maximal condition holds for $(R, \Psi)$-submodules of $\mathbb{F}(A \times A)$ which contain $R$.

The vector space $\mathbb{F}(A \times A) / R$ is isomorphic to the exterior square $\mathbb{F} A \wedge \mathbb{F} A$, which can therefore be given the structure of an $(R, \Psi)$-module. Thus Theorem $\mathrm{D}$ gives the 
following result.

COROLLARY. The maximal condition holds for $(R, \Psi)$-submodules of $\mathbb{F} A \wedge \mathbb{F} A$.

Theorems B, C and D will be proved in Sections 3, 5 and 6, respectively. In Section 2 we show how Theorem A can be derived from Theorems C and D.

\section{The derivation of Theorem $A$}

In this section we assume Theorems $\mathrm{C}$ and $\mathrm{D}$, and we obtain Theorem $\mathrm{A}$ from these results.

One step in the proof of Theorem A is the special case proved in [2]. We could, of course, assume this result, but in order to illustrate our method in a comparatively simple case we first prove this special case.

Let $\mathbf{U}$ and $\mathbf{V}$ be varieties of groups. Let $A$ be a free group in $\mathbf{U}$ on a free generating set $\left\{x_{i}: i \in \mathbb{N}\right\}$ and let $B$ be a free group in $\mathbf{V}$ on a free generating set $\left\{y_{i}^{a}: i \in \mathbb{N}, a \in A\right\}$. For each $i$, the element $y_{i}^{1}$ is also written as $y_{i}$. Each element $a^{\prime}$ of $A$ induces an automorphism of $B$ in which $y_{i}^{a} \mapsto y_{i}^{a a^{\prime}}$ for all $i \in \mathbb{N}, a \in A$. Accordingly we can form the semidirect product $B A$, a split extension of $B$ by $A$ in which the original action of $A$ on $B$ becomes conjugation. We denote this group $B A$ by $F_{\text {split }}(\mathbf{V}, \mathbf{U})$. The group has the following universal property implicit in [14] and straightforward to prove directly.

LEMMA 2.1. Let $G$ be a split extension of a group $B_{1}$ in $\mathrm{V}$ by a group $A_{1}$ in $\mathrm{U}$. Then every pair of mappings $\left\{x_{i}: i \in \mathbb{N}\right\} \rightarrow A_{1},\left\{y_{i}: i \in \mathbb{N}\right\} \rightarrow B_{1}$ extends (uniquely) to a homomorphism $F_{\text {split }}(\mathbf{V}, \mathbf{U}) \rightarrow G$.

LEMMA 2.2. Let $\mathrm{U}$ and $\mathrm{V}$ be locally finite varieties of groups of coprime exponents and write $W=F_{\text {split }}(\mathbf{V}, \mathbf{U})$. Let $\mathbf{S}$ be a subvariety of $\mathrm{VU}$. Then $\mathbf{S}$ is generated by the group $W / \mathbf{S}(W)$, where $\mathbf{S}(W)$ is the verbal subgroup of $W$ corresponding to $\mathbf{S}$.

PROOF. Since $\mathbf{S}$ is locally finite it is generated by the finite groups it contains. By the Schur-Zassenhaus Theorem, each such finite group $G$ is a split extension of a group in $\mathbf{V}$ by a group in $\mathbf{U}$. It follows, by Lemma 2.1 , that $G$ is a homomorphic image of $W / \mathbf{S}(W)$. Therefore $W / \mathbf{S}(W)$ generates $\mathbf{S}$.

LEMMA 2.3. Let $F$ be a relatively free group and let $U$ be an abelian fully invariant subgroup of $F$ of exponent dividing a positive integer $m$. Suppose that $U$ contains an infinite strictly ascending chain of fully invariant subgroups of $F$. Then there exists $a$ prime $p$ dividing $m$ such that $U / U^{p}$ contains an infinite strictly ascending chain of fully invariant subgroups of $F / U^{p}$. 
PROOF. Let $\Omega$ be the set of all endomorphisms of $F$, with $\Omega$ regarded as a set of operators. If $V$ is any fully invariant subgroup of $F$ then, since the endomorphisms of $F / V$ are precisely those induced by elements of $\Omega, F / V$ may be regarded as an $\Omega$-group and the $\Omega$-subgroups of $F / V$ are precisely the fully invariant subgroups of $F / V$, each being of the form $W / V$ for some $\Omega$-subgroup $W$ of $F$ containing $V$. Observe that if $N$ is an $\Omega$-subgroup of $U$ then, since $U$ contains an infinite strictly ascending chain of $\Omega$-subgroups, either $N$ or $U / N$ contains such a chain.

Since $U$ is abelian of exponent dividing $m$, we may write $U$ as a finite direct product $U=U_{1} \times \cdots \times U_{k}$ where each $U_{i}$ is a non-trivial $\Omega$-subgroup of prime-power exponent dividing $m$. By repeated use of the previous observation and isomorphisms of $\Omega$-groups, we find that there exists $i \in\{1, \ldots, k\}$ such that $U / \prod_{j \neq i} U_{j}$ contains an infinite strictly ascending chain of $\Omega$-subgroups. Thus it suffices to prove the lemma in the case where $U$ has exponent $p^{s}$ for some prime $p$ and positive integer $s$. By the same observation applied to the chain $U \geq U^{p} \geq \cdots \geq U^{p^{s}}=\{1\}$, there exists $r \in\{0,1, \ldots, s-1\}$ such that $U^{p^{r}} / U^{p^{r+1}}$ contains an infinite strictly ascending chain of $\Omega$-subgroups. Thus there are $\Omega$-subgroups $W_{1}, W_{2}, \ldots$ of $U$ satisfying

$$
U^{p^{r+1}} \leq W_{1}<W_{2}<\cdots<U^{p^{r}} .
$$

Let $\chi: U \rightarrow U^{p^{r}}$ be the homomorphism defined by $u \chi=u^{p^{\prime}}$ for all $u \in U$. Note that $\chi$ is surjective. Thus $U^{p} \leq W_{1} \chi^{-1}<W_{2} \chi^{-1}<\cdots<U$. It is easily verified that $\chi$ is a homomorphism of $\Omega$-groups. Thus each $W_{i} \chi^{-1}$ is an $\Omega$-group and $W_{1} \chi^{-1} / U^{p}<W_{2} \chi^{-1} / U^{p}<\cdots$ is an infinite strictly ascending chain of fully invariant subgroups of $F / U^{p}$ contained in $U / U^{p}$.

We shall now obtain the finite basis result of [2]. For any variety $\mathbf{V}, F(\mathbf{V})$ denotes the free group of $\mathbf{V}$ of countably infinite rank.

THEOREM 2.4 ([2]). Let $m$ and $n$ be coprime positive integers. Then the subvarieties of $\mathbf{A}_{m} \mathbf{N}_{2, n}$ are finitely based.

PROOF. Since $\mathbf{A}_{m} \mathbf{N}_{2, n}$ is finitely based by [7], it suffices to show that $F\left(\mathbf{A}_{m} \mathbf{N}_{2, n}\right)$ satisfies the maximal condition on fully invariant subgroups. Write $H=F\left(\mathbf{A}_{m} \mathbf{N}_{2, n}\right)$ and $U=\mathbf{N}_{2, n}(H)$. Thus $H / U \cong F\left(\mathbf{N}_{2, n}\right)$. By [11], $H / U$ satisfies the maximal condition on fully invariant subgroups. Thus it suffices to show that the maximal condition holds for fully invariant subgroups of $H$ contained in $U$. By Lemma 2.3, it suffices to show that for each prime $p$ dividing $m$ the maximal condition holds for fully invariant subgroups of $H / U^{p}$ contained in $U / U^{p}$. But $H / U^{p} \cong F\left(\mathbf{A}_{p} \mathbf{N}_{2, n}\right)$, so it suffices to show that the minimal condition holds for subvarieties of $A_{p} \mathbf{N}_{2, n}$ which contain $\mathbf{N}_{2, n}$.

Let $W=F_{\text {split }}\left(\mathbf{A}_{p}, \mathbf{N}_{2, n}\right)$ and write $W=B A$ where $A=\left\langle x_{i}: i \in \mathbb{N}\right\rangle \cong F\left(\mathbf{N}_{2, n}\right)$ and $B=\left\langle y_{i}^{a}: i \in \mathbb{N}, a \in A\right\rangle$. Thus $B$ is free in $\mathbf{A}_{p}$. By Lemma 2.2, the subvarieties 
of $A_{p} \mathbf{N}_{2, n}$ which contain $\mathbf{N}_{2, n}$ are in one-one correspondence with the corresponding verbal subgroups of $W$, and these verbal subgroups are contained in $B$. Thus it suffices to prove that the maximal condition holds for fully invariant subgroups of $W$ contained in $B$.

We can write $B$ additively as a vector space over $\mathbb{F}_{p}$, the field with $p$ elements, and $B$ has basis $\left\{y_{i}^{a}: i \in \mathbb{N}, a \in A\right\}$. Let $T$ be the subspace with basis $\left\{y_{1}^{a}: a \in A\right\}$. There is an $\mathbb{F}_{p}$-space isomorphism $\mu: \mathbb{F}_{p} A \rightarrow T$ satisfying $a \mu=y_{1}^{a}$ for all $a \in A$. Hence we can give $T$ the structure of a left $\mathbb{F}_{p} A$-module in such a way that $\mu$ is a module isomorphism. Let $\Psi$ be the set of all endomorphisms of $A$. By Lemma 2.1, each element $\psi$ of $\Psi$ can be extended to an endomorphism of $W$ by taking $y_{i} \psi=y_{i}$ for each $i$. Thus $\Psi$ acts on $W$. Clearly $T$ is $\Psi$-closed and the map $\mu: \mathbb{F}_{p} A \rightarrow T$ is an isomorphism of $\left(\mathbb{F}_{p} A, \Psi\right)$-modules.

For each $a \in A$, let $\xi_{a}$ be the endomorphism of $W$ satisfying $x_{i} \xi_{a}=x_{i}$ for all $i$, $y_{1} \xi_{a}=y_{1}^{a}$ and $y_{i} \xi_{a}=y_{i}$ for all $i>1$. Clearly $T$ is invariant under each $\xi_{a}$, and $\xi_{a}$ acts on $T$ in the same way as $a$ acts (when $T$ is regarded as a left $\mathbb{F}_{p} A$-module). It follows that if $V$ is a fully invariant subgroup of $W$ then $V \cap T$ is an $\left(\mathbb{F}_{p} A, \Psi\right)$-submodule of $T$.

For each $i, j \in \mathbb{N}$, let $\delta_{i j}$ be the endomorphism of $W$ determined by $x_{k} \delta_{i j}=x_{k}$ for all $k, y_{i} \delta_{i j}=y_{j}$ and $y_{k} \delta_{i j}=1$ for all $k \in \mathbb{N} \backslash\{i\}$. Let $V$ be a fully invariant subgroup of $W$ contained in $B$ and let $v \in V$. Then there exists $r \in \mathbb{N}$ such that $v$ belongs to the span of $\left\{y_{i}^{a}: 1 \leq i \leq r, a \in A\right\}$. We have $v=v \delta_{11} \delta_{11}+v \delta_{21} \delta_{12}+\cdots+v \delta_{r 1} \delta_{1 r}$, where $v \delta_{11}, v \delta_{21}, \ldots, v \delta_{r 1} \in V \cap T$. Thus $V$ is generated as a fully invariant subgroup by $V \cap T$.

Suppose that $V_{1} \leq V_{2} \leq \ldots$ is an ascending chain of fully invariant subgroups of $W$ contained in $B$. Then $V_{1} \cap T \leq V_{2} \cap T \leq \cdots$ is an ascending chain of $\left(\mathbb{F}_{p} A, \Psi\right)$ modules. Hence $\left(V_{1} \cap T\right) \mu^{-1} \leq\left(V_{2} \cap T\right) \mu^{-1} \leq \cdots$ is an ascending chain of $\Psi$-closed left ideals of $\mathbb{F}_{p} A$. By Theorem $\mathrm{C}$, this chain becomes stationary. Therefore, so does $V_{1} \cap T \leq V_{2} \cap T \leq \cdots$, and so does $V_{1} \leq V_{2} \leq \cdots$, which completes the proof of Theorem 2.4.

PROOF OF THEOREM A. Let $m$ and $n$ be coprime positive integers, and write $F=$ $F\left(\mathbf{N}_{2, m} \mathbf{N}_{2, n}\right)$. By [7], $\mathbf{N}_{2, m} \mathbf{N}_{2, n}$ is finitely based. Thus it suffices to show that $F$ satisfies the maximal condition on fully invariant subgroups. Let $U$ be the verbal subgroup of $F$ corresponding to $\mathbf{A}_{m} \mathbf{N}_{2, n}$. Thus $F / U \cong F\left(\mathbf{A}_{m} \mathbf{N}_{2, n}\right)$ and, by Theorem 2.4 , it suffices to show that the maximal condition holds for fully invariant subgroups of $F$ contained in $U$. By Lemma 2.3 it suffices to show that, for each prime $p$ dividing $m$, the maximal condition holds for fully invariant subgroups of $F / U^{p}$ contained in $U / U^{p}$. Let $\mathbf{V}$ be the variety of all groups $G$ such that $G$ is nilpotent of class at most two, $G$ has exponent dividing $m$ and $G^{\prime}$ has exponent dividing $p$. Thus $F / U^{p} \cong F\left(\mathbf{V N}_{2, n}\right)$. It suffices to show that the minimal condition holds for subvarieties of $\mathbf{V N}_{2, n}$ which contain $\mathbf{A}_{m} \mathbf{N}_{2, n}$. 
Let $W=F_{\text {split }}\left(\mathbf{V}, \mathbf{N}_{2, n}\right)$ and write $W=B A$ where $A=\left\langle x_{i}: i \in \mathbb{N}\right\rangle \cong F\left(\mathbf{N}_{2, n}\right)$ and $B=\left\langle y_{i}^{a}: i \in \mathbb{N}, a \in A\right\rangle$. Thus $B$ is free in $\mathbf{V}$. By Lemma 2.2, the subvarieties of $\mathbf{V N}_{2, n}$ which contain $A_{m} \mathbf{N}_{2, n}$ are in one-one correspondence with the corresponding verbal subgroups of $W$, and these verbal subgroups are contained in $B^{\prime}$. Thus it suffices to prove that the maximal condition holds for fully invariant subgroups of $W$ contained in $B^{\prime}$. If $B^{\prime}=\{1\}$ (as occurs when $p=2$ and $m$ is not divisible by 4 ) then the result is trivial. Thus we may assume that $B^{\prime} \neq\{1\}$.

We can write $B^{\prime}$ additively as a vector space over $\mathbb{F}_{p}$ spanned by $\left\{\left[y_{i}^{a}, y_{j}^{a^{\prime}}\right]\right.$ : $\left.i, j \in \mathbb{N}, a, a^{\prime} \in A\right\}$. Let $T_{1}$ be the subspace spanned by $\left\{\left[y_{1}^{a}, y_{1}^{a^{\prime}}\right]: a, a^{\prime} \in A\right\}$ and let $T_{2}$ be the subspace spanned by $\left\{\left[y_{1}^{a}, y_{2}^{a^{\prime}}\right]: a, a^{\prime} \in A\right\}$. Thus $T_{1}$ has basis $\left\{\left[y_{1}^{a}, y_{1}^{a^{\prime}}\right]: a, a^{\prime} \in A, a>a^{\prime}\right\}$, where $>$ is an arbitrary total order on $A$, and $T_{2}$ has basis $\left\{\left[y_{1}^{a}, y_{2}^{a^{\prime}}\right]: a, a^{\prime} \in A\right\}$. Thus there are $\mathbb{F}_{p}$-space isomorphisms $\mu_{1}: \mathbb{F}_{p} A \wedge \mathbb{F}_{p} A \rightarrow T_{1}$ and $\mu_{2}: \mathbb{F}_{p}(A \times A) \rightarrow T_{2}$ satisfying $\left(a \wedge a^{\prime}\right) \mu_{1}=\left[y_{1}^{a}, y_{1}^{a^{\prime}}\right]$ and $\left(a \otimes a^{\prime}\right) \mu_{2}=\left[y_{1}^{a}, y_{2}^{a^{\prime}}\right]$ for all $a, a^{\prime} \in A$. Hence, with $R$ defined as in Section 1 , we can give $T_{1}$ the structure of a left $R$-module and $T_{2}$ the structure of a left $\mathbb{F}_{p}(A \times A)$-module in such a way that $\mu_{1}$ and $\mu_{2}$ are module isomorphisms. Let $\Psi$ be the set of all endomorphisms of $A$. As in the proof of Theorem 2.4, $\Psi$ acts on $W$. Clearly $T_{1}$ and $T_{2}$ are $\Psi$-closed, $\mu_{1}$ is an isomorphism of $(R, \Psi)$-modules, and $\mu_{2}$ is an isomorphism of $\left(\mathbb{F}_{p}(A \times A), \Psi\right)$ modules.

For $a \in A$, let $\xi_{a}$ be the endomorphism of $W$ satisfying $x_{i} \xi_{a}=x_{i}$ for all $i$, $y_{1} \xi_{a}=y_{1}^{a}$ and $y_{i} \xi_{a}=y_{i}$ for all $i>1$. For $a, a^{\prime} \in A$, let $\xi_{a+a^{\prime}}$ be the endomorphism of $W$ satisfying $x_{i} \xi_{a+a^{\prime}}=x_{i}$ for all $i, y_{1} \xi_{a+a^{\prime}}=y_{1}^{a} y_{1}^{a^{\prime}}$ and $y_{i} \xi_{a+a^{\prime}}=y_{i}$ for all $i>1$. Thus $T_{1}$ is invariant under each $\xi_{a}$ and under each $\xi_{a+a^{\prime}}$. Furthermore, $\xi_{a}$ acts on $T_{1}$ in the same way as $a \otimes a$ acts, while $\xi_{a+a^{\prime}}$ acts on $T_{1}$ in the same way as $\left(a+a^{\prime}\right) \otimes\left(a+a^{\prime}\right)$ acts. It is easily verified that $R$ is spanned by the elements $a \otimes a$ and $\left(a+a^{\prime}\right) \otimes\left(a+a^{\prime}\right)$ for $a, a^{\prime} \in A$. It follows that if $V$ is a fully invariant subgroup of $W$ then $V \cap T_{1}$ is an $(R, \Psi)$-submodule of $T_{1}$.

For $a, a^{\prime} \in A$, let $\xi_{a, a^{\prime}}$ be the endomorphism of $W$ determined by $x_{i} \xi_{a, a^{\prime}}=x_{i}$ for all $i, y_{1} \xi_{a, a^{\prime}}=y_{1}^{a}, y_{2} \xi_{a, a^{\prime}}=y_{2}^{a^{\prime}}$ and $y_{i} \xi_{a, a^{\prime}}=y_{i}$ for all $i>2$. Clearly $T_{2}$ is invariant under each $\xi_{a, a^{\prime}}$. Furthermore, $\xi_{a, a^{\prime}}$ acts on $T_{2}$ in the same way as $a \otimes a^{\prime}$ acts. It follows that if $V$ is a fully invariant subgroup of $W$ then $V \cap T_{2}$ is an $\left(\mathbb{F}_{p}(A \times A), \Psi\right)$-submodule of $T_{2}$.

For each $i, j \in \mathbb{N}$, let $\delta_{i j}$ be the endomorphism of $W$ determined by $x_{k} \delta_{i j}=x_{k}$ for all $k, y_{i} \delta_{i j}=y_{j}$ and $y_{k} \delta_{i j}=1$ for all $k \in \mathbb{N} \backslash\{i\}$. For each $i, j, i^{\prime}, j^{\prime} \in \mathbb{N}$ with $i \neq j$, let $\varepsilon_{i i^{\prime}, j j^{\prime}}$ be the endomorphism of $W$ determined by $x_{k} \varepsilon_{i i^{\prime}, j j^{\prime}}=x_{k}$ for all $k$, $y_{i} \varepsilon_{i i^{\prime}, j j^{\prime}}=y_{i^{\prime}}, y_{j} \varepsilon_{i i^{\prime}, j j^{\prime}}=y_{j^{\prime}}$ and $y_{k} \varepsilon_{i i^{\prime}, j j^{\prime}}=1$ for all $k \in \mathbb{N} \backslash\{i, j\}$.

Let $V$ be a fully invariant subgroup of $W$ contained in $B^{\prime}$ and let $v \in V$. Then, for some $r \in \mathbb{N}$, we can write $v=v_{1}+v_{2}$ where $v_{1}$ is in the span of $\left\{\left[y_{i}^{a}, y_{i}^{a^{\prime}}\right]: 1 \leq i \leq\right.$ $\left.r, a, a^{\prime} \in A\right\}$ and $v_{2}$ is in the span of $\left\{\left[y_{i}^{a}, y_{j}^{a^{\prime}}\right]: 1 \leq i<j \leq r, a, a^{\prime} \in A\right\}$. Then it is 
easily verified that $v_{1}=\sum_{i} v \delta_{i 1} \delta_{1 i}$ and

$$
v-v_{1}=v_{2} \doteq \sum_{\substack{i, j \\ 1 \leq i<j \leq r}} v_{2} \varepsilon_{i 1, j 2} \varepsilon_{1 i, 2 j} .
$$

Here $v \delta_{i 1} \in V \cap T_{1}$ for all $i$ and $v_{2} \varepsilon_{i 1, j 2} \in V \cap T_{2}$ for all $i, j$. It follows that $V$ is generated as a fully invariant subgroup by $\left(V \cap T_{1}\right) \cup\left(V \cap T_{2}\right)$.

Suppose that $V_{1} \leq V_{2} \leq \cdots$ is an ascending chain of fully invariant subgroups of $W$ contained in $B^{\prime}$. Then $V_{1} \cap T_{1} \leq V_{2} \cap T_{1} \leq \cdots$ is an ascending chain of $(R, \Psi)$-submodules of $T_{1}$ while $V_{1} \cap T_{2} \leq V_{2} \cap T_{2} \leq \cdots$ is an ascending chain of $\left(\mathbb{F}_{p}(A \times A), \Psi\right)$-submodules of $T_{2}$. Hence $\left(V_{1} \cap T_{1}\right) \mu_{1}^{-1} \leq\left(V_{2} \cap T_{1}\right) \mu_{1}^{-1} \leq \cdots$ is an ascending chain of $(R, \Psi)$-submodules of $\mathbb{F}_{p} A \wedge \mathbb{F}_{p} A$ and

$$
\left(V_{1} \cap T_{2}\right) \mu_{2}^{-1} \leq\left(V_{2} \cap T_{2}\right) \mu_{2}^{-1} \leq \cdots
$$

is an ascending chain of $\Psi$-closed left ideals of $\mathbb{F}_{p}(A \times A)$. By Theorem $\mathrm{C}$ and the Corollary to Theorem $D$, both of the last two chains become stationary. Hence $\left(V_{1} \cap T_{1}\right) \cup\left(V_{1} \cap T_{2}\right) \leq\left(V_{2} \cap T_{1}\right) \cup\left(V_{2} \cap T_{2}\right) \leq \cdots$ becomes stationary. Therefore $V_{1} \leq V_{2} \leq \cdots$ becomes stationary, which proves Theorem $\mathrm{A}$.

\section{Bilinear forms}

Let $K$ be a non-zero, finite, commutative and associative ring, with identity element 1 . Unless otherwise stated all $K$-modules are finitely generated (therefore finite). Let $S$ be a $K$-module. An $S$-form is a pair $(V, \theta)$ consisting of a non-zero free $K$-module $V$ and a $K$-bilinear map $\theta: V \times V \rightarrow S$. A $K$-linear map $\xi: V \rightarrow V^{\prime}$, where $(V, \theta)$ and $\left(V^{\prime}, \theta^{\prime}\right)$ are $S$-forms, is said to be a homomorphism of $S$-forms if $\theta\left(v_{1}, v_{2}\right)=\theta^{\prime}\left(v_{1} \xi, v_{2} \xi\right)$ for all $v_{1}, v_{2} \in V$. We write $\xi:(V, \theta) \rightarrow\left(V^{\prime}, \theta^{\prime}\right)$. The terms isomorphism and monomorphism are defined in the obvious way. We define a quasi-order $\preccurlyeq$ on the set of all $S$-forms by defining $(V, \theta) \preccurlyeq\left(V^{\prime}, \theta^{\prime}\right)$ if there is a monomorphism $\xi:(V, \theta) \rightarrow\left(V^{\prime}, \theta^{\prime}\right)$. The main result of this section is the following.

THEOREM B. The set of all $S$-forms is well-quasi-ordered under the relation $\preccurlyeq$.

Let $(V, \theta)$ be an $S$-form. For any subset $U$ of $V$ we define $P(U)$ to be the subset of $S \oplus S$ given by $P(U)=\left\{\left(\theta\left(v_{1}, v_{2}\right), \theta\left(v_{2}, v_{1}\right)\right): v_{1}, v_{2} \in U\right\}$, and we define $Q(U) \subseteq S$ by $Q(U)=\{\theta(v, v): v \in U\}$. Also, for $U, U^{\prime} \subseteq V$ we define $\theta\left(U, U^{\prime}\right) \subseteq S$ by $\theta\left(U, U^{\prime}\right)=\left\{\theta\left(u, u^{\prime}\right): u \in U, u^{\prime} \in U^{\prime}\right\}$. Subsets $U$ and $U^{\prime}$ are said to be orthogonal if $\theta\left(U, U^{\prime}\right)=\theta\left(U^{\prime}, U\right)=\{0\}$. 
LEMMA 3.1. Let $V$ be a free $K$-module and let $v_{1}, \ldots, v_{l} \in V$. Then there are free $K$-submodules $U_{1}, U_{2}$ of $V$ such that $V=U_{1} \oplus U_{2}, \operatorname{rank}\left(U_{1}\right) \leq|K| l$, and $v_{1}, \ldots, v_{l} \in U_{1}$.

ProOF. Take elements $x_{1}, \ldots, x_{m}$ of $V$ where $m$ is minimal such that $\left\{x_{1}, \ldots, x_{m}\right\}$ is contained in a $K$-basis of $V$ and $v_{1}$ belongs to the submodule $\left\langle x_{1}, \ldots, x_{m}\right\rangle$. Write $v_{1}=\sum_{i=1}^{m} \alpha_{i} x_{i}$ where each $\alpha_{i}$ is an element of $K$. If $m>|K|$ then there exist distinct $j, k \in\{1, \ldots, m\}$ such that $\alpha_{j}=\alpha_{k}$ and we may replace $x_{j}$ and $x_{k}$ by $x_{j}+x_{k}$, contrary to the minimality of $m$. Thus $m \leq|K|$. Let $W$ be a free $K$-submodule of $V$ such that $V=\left\langle x_{1}, \ldots, x_{m}\right\rangle \oplus W$ and, for $i=2, \ldots, l$, write $v_{i}=v_{i}^{\prime}+w_{i}$ where $v_{i}^{\prime} \in\left\langle x_{1}, \ldots, x_{m}\right\rangle$ and $w_{i} \in W$. The result follows by applying an inductive argument to $w_{2}, \ldots, w_{l}$ in $W$.

LEMMA 3.2. Let $(V, \theta)$ be an $S$-form. Suppose that $W$ is a free $K$-submodule of $V$ and let $v_{1}, \ldots, v_{l} \in V$. Then there are free $K$-submodules $W_{1}, W_{2}$ of $W$ such that $W=W_{1} \oplus W_{2}, \operatorname{rank}\left(W_{1}\right) \leq 2|S| l$ and $W_{2}$ is orthogonal to $\left\{v_{1}, \ldots, v_{l}\right\}$.

PROOF. We assume that $l=1$ since the general case follows easily. We shall find free submodules $U_{1}, U_{2}$ of $W$ such that $W=U_{1} \oplus U_{2}, \operatorname{rank}\left(U_{1}\right) \leq|S|$ and $\theta\left(\left\{v_{1}\right\}, U_{2}\right)=\{0\}$. A similar argument gives $U_{2}=U^{\prime} \oplus U^{\prime \prime}$ with $\operatorname{rank}\left(U^{\prime}\right) \leq|S|$ and $\theta\left(U^{\prime \prime},\left\{v_{1}\right\}\right)=\{0\}$. The result follows with $W_{1}=U_{1} \oplus U^{\prime}$ and $W_{2}=U^{\prime \prime}$.

Take basis elements $x_{1}, \ldots, x_{m}$ of $W$ where $m$ is maximal subject to $\theta\left(v_{1}, x_{i}\right)=0$ for $i=1, \ldots, m$. Let $\left\{x_{1}, \ldots, x_{d}\right\}$ be a basis of $W$ containing $\left\{x_{1}, \ldots, x_{m}\right\}$. If $d-m>$ $|S|$ then there exist distinct $j, k \in\{m+1, \ldots, d\}$ such that $\theta\left(v_{1}, x_{j}\right)=\theta\left(v_{1}, x_{k}\right)$ and we may extend $\left\{x_{1}, \ldots, x_{m}\right\}$ to $\left\{x_{1}, \ldots, x_{m}, x_{j}-x_{k}\right\}$, contrary to the maximality of $m$. Thus $d-m \leq|S|$ and we may take $U_{1}=\left\langle x_{m+1}, \ldots, x_{d}\right\rangle, U_{2}=\left\langle x_{1}, \ldots, x_{m}\right\rangle$.

Let $N$ be a positive integer and define $N^{[i]}$, for each non-negative integer $i$, by $N^{[0]}=0$ and $N^{[i]}=N+N^{2}+\cdots+N^{i}$ for $i>0$. Let $(V, \theta)$ be an $S$-form and let $\left\{x_{1}, \ldots, x_{d}\right\}$ be a $K$-basis of $V$. We shall assume, in such notation, that the elements $x_{i}$ are distinct (that is, $d=\operatorname{rank}(V)$ ) and that the basis is ordered as shown, corresponding to the ordered $d$-tuple $\left(x_{1}, \ldots, x_{d}\right)$. Let $m$ be the nonnegative integer which satisfies $N^{[m]}<d \leq N^{[m+1]}$ and write $V_{1}=\left\langle x_{1}, \ldots, x_{N^{[1]}}\right\rangle, \ldots$, $V_{m}=\left\langle x_{N^{[m-1]}+1}, \ldots, x_{N^{[m]}}\right\rangle, V_{m+1}=\left\langle x_{N^{[m]}+1}, \ldots, x_{d}\right\rangle$. Thus $\operatorname{rank}\left(V_{i}\right)=N^{i}$ for $i=1, \ldots, m$ and $0<\operatorname{rank}\left(V_{m+1}\right) \leq N^{m+1}$. For $i=1, \ldots, m+1$, write $V_{i}^{+}=$ $V_{i} \oplus \cdots \oplus V_{m+1}$. We say that $(V, \theta)$ is $N$-regular with respect to the ordered basis $\left\{x_{1}, \ldots, x_{d}\right\}$ if $P\left(V_{i}\right)=P\left(V_{i}^{+}\right)$for $i=1, \ldots, m+1, Q\left(V_{i}\right)=Q\left(V_{i}^{+}\right)$for $i=$ $1, \ldots, m+1$, and $V_{i-1}$ and $V_{i+1}^{+}$are orthogonal for $i=2, \ldots, m$. A decomposition $V=V_{1} \oplus \cdots \oplus V_{m+1}$ with these properties, which is obtained from some ordered basis in the way described, is called an $N$-regular decomposition of $V$. Note that $V_{i}$ 
and $V_{j}$ are orthogonal whenever $|i-j| \geq 2$. Also $P\left(V_{1}\right) \supseteq P\left(V_{2}\right) \supseteq \cdots \supseteq P\left(V_{m+1}\right)$ and $Q\left(V_{1}\right) \supseteq Q\left(V_{2}\right) \supseteq \cdots \supseteq Q\left(V_{m+1}\right)$.

LEMMA 3.3. Let $N \geq|K|\left(2|S|^{2}+|S|\right)$. Then every $S$-form is $N$-regular with respect to some basis.

PROOF. Write $s=|S|$. Let $(V, \theta)$ be an $S$-form. Let $d=\operatorname{rank}(V)$ and define $m$ by $N^{[m]}<d \leq N^{[m+1]}$. Suppose we can find free modules $V_{1}^{+}, V_{1}, V_{2}^{+}, V_{2}, \ldots, V_{m}^{+}$, $V_{m}, V_{m+1}^{+}$with the following properties: $V_{1}^{+}=V$; for $i=1, \ldots, m, V_{i}^{+}=V_{i} \oplus V_{i+1}^{+}$, $\operatorname{rank}\left(V_{i}\right)=N^{i}, P\left(V_{i}\right)=P\left(V_{i}^{+}\right)$and $Q\left(V_{i}\right)=Q\left(V_{i}^{+}\right)$; and, for $i=2, \ldots, m, V_{i-1}$ and $V_{i+1}^{+}$are orthogonal. Then, taking $V_{m+1}=V_{m+1}^{+}$, we see that $V=V_{1} \oplus \cdots \oplus V_{m+1}$ and $(V, \theta)$ is $N$-regular with respect to a basis of $V$ composed of bases of $V_{1}, \ldots, V_{m+1}$. We construct the required free modules inductively.

First define $V_{1}^{+}=V$. If $\operatorname{rank}\left(V_{1}^{+}\right) \leq N$ then $m=0$ and we have finished. So suppose that $\operatorname{rank}\left(V_{1}^{+}\right)>N$. Since $\left|P\left(V_{1}^{+}\right)\right| \leq s^{2}$ and $\left|Q\left(V_{1}^{+}\right)\right| \leq s$ we can choose elements $v_{1}, \ldots, v_{2 s^{2}+s}$ of $V_{1}^{+}$(not necessarily distinct) such that

$$
\begin{aligned}
\left\{\left(\theta\left(v_{2 i-1}, v_{2 i}\right), \theta\left(v_{2 i}, v_{2 i-1}\right)\right): i=1, \ldots, s^{2}\right\} & =P\left(V_{1}^{+}\right), \\
\left\{\theta\left(v_{i}, v_{i}\right): i=2 s^{2}+1, \ldots, 2 s^{2}+s\right\} & =Q\left(V_{1}^{+}\right) .
\end{aligned}
$$

By Lemma 3.1, we can find free submodules $U_{1}$ and $U_{2}$ of $V_{1}^{+}$such that $V_{1}^{+}=U_{1} \oplus U_{2}$, $v_{1}, \ldots, v_{2 s^{2}+s} \in U_{1}$ and $\operatorname{rank}\left(U_{1}\right) \leq|K|\left(2 s^{2}+s\right) \leq N$. Choose free modules $V_{1}$ and $V_{2}^{+}$such that $V_{1}^{+}=V_{1} \oplus V_{2}^{+}, \operatorname{rank}\left(V_{1}\right)=N$ and $V_{1} \supseteq U_{1}$. By the choice of $v_{1}, \ldots, v_{2 s^{2}+s}$, we have $P\left(V_{1}\right)=P\left(V_{1}^{+}\right)$and $Q\left(V_{1}\right)=Q\left(V_{1}^{+}\right)$.

Suppose that for some $k$ with $1 \leq k \leq m$ we have found free modules $V_{1}^{+}, V_{1}, V_{2}^{+}$, $\ldots, V_{k}, V_{k+1}^{+}$with the required properties for these modules. If $\operatorname{rank}\left(V_{k+1}^{+}\right) \leq N^{k+1}$ then $m=k$ and we have finished. So suppose that $\operatorname{rank}\left(V_{k+1}^{+}\right)>N^{k+1}$. By the method used in the first part of the proof we may find free submodules $U$ and $W$ of $V_{k+1}^{+}$such that $V_{k+1}^{+}=U \oplus W, P(U)=P\left(V_{k+1}^{+}\right), Q(U)=Q\left(V_{k+1}^{+}\right)$and $\operatorname{rank}(U)=N$. By Lemma 3.2, there are free submodules $W_{1}$ and $W_{2}$ of $W$ such that $W=W_{1} \oplus W_{2}, W_{2}$ and $V_{k}$ are orthogonal and $\operatorname{rank}\left(W_{1}\right) \leq 2 s N^{k}$. Then

$$
\operatorname{rank}\left(U \oplus W_{1}\right) \leq N+2 s N^{k} \leq(1+2 s) N^{k} \leq N^{k+1} .
$$

Choose free modules $V_{k+1}$ and $V_{k+2}^{+}$such that $V_{k+1}^{+}=V_{k+1} \oplus V_{k+2}^{+}$, $\operatorname{rank}\left(V_{k+1}\right)=N^{k+1}$, $V_{k+1} \supseteq U \oplus W_{1}$ and $V_{k+2}^{+} \subseteq W_{2}$. Then $V_{k+1}$ and $V_{k+2}^{+}$have the required properties.

LEMMA 3.4. Let $(V, \theta)$ be an $S$-form which has an $N$-regular decomposition $V=$ $V_{1} \oplus \cdots \oplus V_{m+1}$.

(i) Let $k \in\{1, \ldots, m-1\}$. Suppose that $P\left(V_{k}\right)=P\left(V_{k+2}\right)$ and $Q\left(V_{k}\right)=$ $Q\left(V_{k+2}\right)$. Then $P\left(V_{k}\right)$ is an additive subgroup of $S \oplus S$ and $Q\left(V_{k}\right)$ is an additive subgroup of $S$. 
(ii) Let $c$ be a positive integer and let $r(1)$ and $r(2)$ be integers such that $1 \leq$ $r(1)<r(2) \leq m+1$. Suppose that

$$
\begin{aligned}
& P\left(V_{r(1)}\right)=P\left(V_{r(1)+1}\right)=\cdots=P\left(V_{r(2)}\right)=P \subseteq S \oplus S, \\
& Q\left(V_{r(1)}\right)=Q\left(V_{r(1)+1}\right)=\cdots=Q\left(V_{r(2)}\right)=Q \subseteq S,
\end{aligned}
$$

and $r(2)-r(1) \geq c(c+1)+2$. Write $W=V_{r(1)+2} \oplus \cdots \oplus V_{r(2)-2}$. For all $i, j \in\{1, \ldots, c\}$ with $i<j$ let $p_{i j} \in P$ and for all $i \in\{1, \ldots, c\}$ let $q_{i} \in Q$. Then there exist $w_{1}, \ldots, w_{c} \in W$ such that $\left(\theta\left(w_{i}, w_{j}\right), \theta\left(w_{j}, w_{i}\right)\right)=p_{i j}$, for all $i, j \in\{1, \ldots, c\}$ with $i<j$, and $\theta\left(w_{i}, w_{i}\right)=q_{i}$, for all $i \in\{1, \ldots, c\}$.

PROOF. (i) Let $p, p^{\prime} \in P\left(V_{k}\right)$. Then there exist $v, w \in V_{k}$ and $v^{\prime}, w^{\prime} \in V_{k+2}$ such that $(\theta(v, w), \theta(w, v))=p$ and $\left(\theta\left(v^{\prime}, w^{\prime}\right), \theta\left(w^{\prime}, v^{\prime}\right)\right)=p^{\prime}$. Write $V_{k}^{+}=$ $V_{k} \oplus \cdots \oplus V_{m+1}$. Since $V_{k}$ and $V_{k+2}$ are orthogonal,

$$
p+p^{\prime}=\left(\theta\left(v+v^{\prime}, w+w^{\prime}\right), \theta\left(w+w^{\prime}, v+v^{\prime}\right)\right) \in P\left(V_{k}^{+}\right)=P\left(V_{k}\right) .
$$

Hence, since $P\left(V_{k}\right)$ is finite, it is a group. Similarly $Q\left(V_{k}\right)$ is a group.

(ii) By (i), $P$ and $Q$ are additive groups. There are $c(c+1) / 2$ modules in the set $\left\{V_{r(1)+2}, V_{r(1)+4}, \ldots, V_{r(1)+c(c+1)}\right\}$ and so these modules can be relabelled as $U_{i}$ for $1 \leq i \leq c$ and $U_{i j}$ for $1 \leq i<j \leq c$. These modules are pairwise orthogonal submodules of $W$ such that $P\left(U_{i}\right)=P\left(U_{i j}\right)=P$ and $Q\left(U_{i}\right)=Q\left(U_{i j}\right)=Q$ for all $i, j$. For $i, j \in\{1, \ldots, c\}$ with $i<j$ choose $u_{i j}, v_{i j} \in U_{i j}$ such that

$$
\left(\theta\left(u_{i j}, v_{i j}\right), \theta\left(v_{i j}, u_{i j}\right)\right)=p_{i j} .
$$

Then for each $i \in\{1, \ldots, c\}$ choose $u_{i} \in U_{i}$ such that

$$
\theta\left(u_{i}, u_{i}\right)=q_{i}-\sum_{j: j>i} \theta\left(u_{i j}, u_{i j}\right)-\sum_{j: j<i} \theta\left(v_{j i}, v_{j i}\right)
$$

Finally, for $i=1, \ldots, c$, define $w_{i}=u_{i}+\sum_{j: j>i} u_{i j}+\sum_{j: j<i} v_{j i}$. It is easy to check that these elements have the required properties.

For each $S$-form $(V, \theta)$ we need to fix an ordered basis of $V$. Thus we define an $S$-triple to be a triple $(V, \theta, X)$ where $(V, \theta)$ is an $S$-form and $X$ is an ordered basis of $V$.

Let $(V, \theta, X)$ and $\left(V^{\prime}, \theta^{\prime}, X^{\prime}\right)$ be $S$-triples, where $\operatorname{rank}(V)=d, \operatorname{rank}\left(V^{\prime}\right)=d^{\prime}$, $X=\left\{x_{1}, \ldots, x_{d}\right\}$ and $X^{\prime}=\left\{x_{1}^{\prime}, \ldots, x_{d^{\prime}}^{\prime}\right\}$. We say that $(V, \theta, X)$ and $\left(V^{\prime}, \theta^{\prime}, X^{\prime}\right)$ are isomorphic if $d=d^{\prime}$ and there is an $S$-form isomorphism $\xi:(V, \theta) \rightarrow\left(V^{\prime}, \theta^{\prime}\right)$ such that $x_{i} \xi=x_{i}^{\prime}$ for $i=1, \ldots, d$. We write $(V, \theta, X) \preccurlyeq\left(V^{\prime}, \theta^{\prime}, X^{\prime}\right)$ if there is a 
one-one order-preserving map $\phi:\{1, \ldots, d\} \rightarrow\left\{1, \ldots, d^{\prime}\right\}$ together with an $S$-form homomorphism $\xi:(V, \theta) \rightarrow\left(V^{\prime}, \theta^{\prime}\right)$ such that, for $i=1, \ldots, d$,

$$
x_{i} \xi=x_{i \phi}^{\prime}+z_{i}, \quad \text { for some } z_{i} \in\left\langle x_{1}^{\prime}, x_{2}^{\prime}, \ldots, x_{i \phi-1}^{\prime}\right\rangle
$$

Clearly $\preccurlyeq$ is a quasi-order on the set of all $S$-triples. Also, if $\xi$ satisfies (3.1) then $\xi$ is a monomorphism. Hence $(V, \theta, X) \preccurlyeq\left(V^{\prime}, \theta^{\prime}, X^{\prime}\right)$ implies $(V, \theta) \preccurlyeq\left(V^{\prime}, \theta^{\prime}\right)$. An $S$-triple $(V, \theta, X)$ is said to be $N$-regular if $(V, \theta)$ is $N$-regular with respect to $X$.

PROPOSITION 3.5. The set of all $N$-regular S-triples is well-quasi-ordered under the relation $\preccurlyeq$.

ProOF. Let $Y^{(1)}, Y^{(2)}, Y^{(3)}, \ldots$ be an infinite sequence of $N$-regular $S$-triples. It suffices to show that there exist integers $i$ and $j$ with $i<j$ such that $Y^{(i)} \preccurlyeq Y^{(j)}$. For each $i$, let $Y^{(i)}=\left(V^{(i)}, \theta^{(i)}, X^{(i)}\right)$ where $V^{(i)}$ has $N$-regular decomposition $V_{1}^{(i)} \oplus$ $\cdots \oplus V_{m(i)+1}^{(i)}, d(i)=\operatorname{rank}\left(V^{(i)}\right)$ and $X^{(i)}=\left\{x_{1}^{(i)}, \ldots, x_{d(i)}^{(i)}\right\}$. If $\{m(1), m(2), \ldots\}$ is bounded then there are only finitely many isomorphism types in the sequence $Y^{(1)}, Y^{(2)}, Y^{(3)}, \ldots$ and the result is clear. Thus we assume that $\{m(1), m(2), \ldots\}$ is unbounded. By passing to an infinite subsequence we may assume that $m(i) \geq 1$ for all $i \geq 1$. There are only finitely many possibilities for the values $\theta^{(i)}\left(x_{j}^{(i)}, x_{k}^{(i)}\right)$ for $j, k \in\left\{1, \ldots, N^{[1]}\right\}$. Thus, by passing to an infinite subsequence, we may assume that, for all $j, k \in\left\{1, \ldots, N^{[1]}\right\}$, the value $\theta^{(i)}\left(x_{j}^{(i)}, x_{k}^{(i)}\right)$ is independent of $i$. Then, by passing to an infinite subsequence, we may assume that $m(i) \geq 2$ for all $i \geq 2$ and that, for all $j, k \in\left\{1, \ldots, N^{[2]}\right\}$, the value $\theta^{(i)}\left(x_{j}^{(i)}, x_{k}^{(i)}\right)$ is independent of $i$ for all $i \geq 2$. Continuing in this way we may pass to an infinite subsequence with the following property for all $n \in \mathbb{N}$ :

$$
m(i) \geq n \text { for all } i \geq n \text { and, }
$$

for all $j, k \in\left\{1, \ldots, N^{[n]}\right\}, \theta^{(i)}\left(x_{j}^{(i)}, x_{k}^{(i)}\right)$ is independent of $i$ for all $i \geq n$.

Let $\bar{V}$ be a free $K$-module with countably infinite basis $\bar{X}=\left\{\bar{x}_{1}, \bar{x}_{2}, \ldots\right\}$. Define a $K$-bilinear map $\bar{\theta}: \bar{V} \times \bar{V} \rightarrow S$ by taking $\bar{\theta}\left(\bar{x}_{j}, \bar{x}_{k}\right)$ to be the limiting value of $\theta^{(i)}\left(x_{j}^{(i)}, x_{k}^{(i)}\right)$. Furthermore, for each positive integer $n$, let $P_{n}$ and $Q_{n}$ be the limiting values of $P\left(V_{n}^{(i)}\right)$ and $Q\left(V_{n}^{(i)}\right)$, respectively. Since $P_{1} \supseteq P_{2} \supseteq \cdots$ and $Q_{1} \supseteq Q_{2} \supseteq \cdots$, there exist $P \subseteq S \oplus S, Q \subseteq S$, and a positive integer $r$, such that $P_{r}=P_{r+1}=\cdots=P$ and $Q_{r}=Q_{r+1}=\cdots=Q$. By Lemma 3.4, $P$ and $Q$ are additive groups.

For each $i$, let $r(i)$ be the largest integer belonging to $\{1, \ldots, m(i)\}$ such that $\theta^{(i)}\left(x_{j}^{(i)}, x_{k}^{(i)}\right)=\bar{\theta}\left(\bar{x}_{j}, \bar{x}_{k}\right)$ for all $j, k \in\left\{1, \ldots, N^{[r(i)]}\right\}$. By construction, the set $\{r(1), r(2), \ldots\}$ is unbounded. Hence, by passing to an infinite subsequence, we may 
assume that $r \leq r(1)<r(2)<\cdots$. Let

$$
\begin{aligned}
& a(i)=N^{[r(i)-1]}=\operatorname{rank}\left(V_{1}^{(i)} \oplus \cdots \oplus V_{r(i)-1}^{(i)}\right) \\
& b(i)=N^{[r(i)]}=\operatorname{rank}\left(V_{1}^{(i)} \oplus \cdots \oplus V_{r(i)}^{(i)}\right)=a(i)+N^{r(i)}
\end{aligned}
$$

We may pass to an infinite subsequence so that, for each $i$, we have

$$
\begin{aligned}
d(i)-a(i) & \leq d(i+1)-a(i+1) \quad \text { and } \\
r(i+1)-r(i) & \geq(d(i)-a(i))(d(i)-a(i)+1)+2 .
\end{aligned}
$$

We now focus on $Y^{(1)}$ and $Y^{(2)}$ and show that $Y^{(1)} \preccurlyeq Y^{(2)}$. By the choice of $r(1)$ and $r(2)$, we have

$$
\begin{aligned}
& P\left(V_{r(1)}^{(1)}\right)=P\left(V_{r(1)}^{(2)}\right)=P\left(V_{r(1)+1}^{(2)}\right)=\cdots=P\left(V_{r(2)}^{(2)}\right)=P, \\
& Q\left(V_{r(1)}^{(1)}\right)=Q\left(V_{r(1)}^{(2)}\right)=Q\left(V_{r(1)+1}^{(2)}\right)=\cdots=Q\left(V_{r(2)}^{(2)}\right)=Q,
\end{aligned}
$$

and

$$
\theta^{(1)}\left(x_{i}^{(1)}, x_{j}^{(1)}\right)=\theta^{(2)}\left(x_{i}^{(2)}, x_{j}^{(2)}\right) \text { for all } i, j \in\{1, \ldots, b(1)\}
$$

Since $a(1)<a(2)$ and $d(1)-a(1) \leq d(2)-a(2)$ there exists a one-one orderpreserving map $\phi:\{1, \ldots, d(1)\} \rightarrow\{1, \ldots, d(2)\}$ such that $i \phi=i$ for $i=1, \ldots, a(1)$ and $\{a(1)+1, \ldots, d(1)\} \phi \subseteq\{a(2)+1, \ldots, d(2)\}$.

Write $W=V_{r(1)+2}^{(2)} \oplus \cdots \oplus V_{r(2)-2}^{(2)}$ as in Lemma 3.4. Note that, for $i \in\{a(1)+$ $1, \ldots, d(1)\}$

$$
\theta^{(1)}\left(x_{i}^{(1)}, x_{i}^{(1)}\right) \in Q\left(V_{r(1)}^{(1)} \oplus \cdots \oplus V_{m(1)+1}^{(1)}\right)=Q\left(V_{r(1)}^{(1)}\right)=Q
$$

and

$$
\theta^{(2)}\left(x_{i \phi}^{(2)}, x_{i \phi}^{(2)}\right) \in Q\left(V_{r(2)}^{(2)} \oplus \cdots \oplus V_{m(2)+1}^{(2)}\right)=Q\left(V_{r(2)}^{(2)}\right)=Q
$$

Similarly,

$$
\left(\theta^{(1)}\left(x_{i}^{(1)}, x_{j}^{(1)}\right), \theta^{(1)}\left(x_{j}^{(1)}, x_{i}^{(1)}\right)\right) \in P, \quad\left(\theta^{(2)}\left(x_{i \phi}^{(2)}, x_{j \phi}^{(2)}\right), \theta^{(2)}\left(x_{j \phi}^{(2)}, x_{i \phi}^{(2)}\right)\right) \in P
$$

for all $i, j \in\{a(1)+1, \ldots, d(1)\}$ with $i<j$. Hence, by Lemma 3.4, we can choose elements $w_{a(1)+1}, \ldots, w_{d(1)}$ of $W$ satisfying

$$
\theta^{(2)}\left(w_{i}, w_{i}\right)= \begin{cases}-\theta^{(2)}\left(x_{i \phi}^{(2)}, x_{i \phi}^{(2)}\right) & \text { for } i \in\{a(1)+1, \ldots, b(1)\} \\ \theta^{(1)}\left(x_{i}^{(1)}, x_{i}^{(1)}\right)-\theta^{(2)}\left(x_{i \phi}^{(2)}, x_{i \phi}^{(2)}\right) & \text { for } i \in\{b(1)+1, \ldots, d(1)\}\end{cases}
$$


and

$$
\begin{aligned}
& \left(\theta^{(2)}\left(w_{i}, w_{j}\right), \theta^{(2)}\left(w_{j}, w_{i}\right)\right) \\
& \quad=\left\{\begin{array}{c}
\left(\theta^{(1)}\left(x_{i}^{(1)}, x_{j}^{(1)}\right), \theta^{(1)}\left(x_{j}^{(1)}, x_{i}^{(1)}\right)\right)-\left(\theta^{(2)}\left(x_{i \phi}^{(2)}, x_{j \phi}^{(2)}\right), \theta^{(2)}\left(x_{j \phi}^{(2)}, x_{i \phi}^{(2)}\right)\right) \\
\quad \text { for } i<j \text { with } i \in\{a(1)+1, \ldots, b(1)\}, j \in\{b(1)+1, \ldots, d(1)\} \\
-\left(\theta^{(2)}\left(x_{i \phi}^{(2)}, x_{j \phi}^{(2)}\right), \theta^{(2)}\left(x_{j \phi}^{(2)}, x_{i \phi}^{(2)}\right)\right) \\
\text { for } i<j \text { with } i, j \in\{a(1)+1, \ldots, b(1)\} \\
\left(\theta^{(1)}\left(x_{i}^{(1)}, x_{j}^{(1)}\right), \theta^{(1)}\left(x_{j}^{(1)}, x_{i}^{(1)}\right)\right)-\left(\theta^{(2)}\left(x_{i \phi}^{(2)}, x_{j \phi}^{(2)}\right), \theta^{(2)}\left(x_{j \phi}^{(2)}, x_{i \phi}^{(2)}\right)\right) \\
\text { for } i<j \text { with } i, j \in\{b(1)+1, \ldots, d(1)\} .
\end{array}\right.
\end{aligned}
$$

Then we define a $K$-linear map $\xi: V^{(1)} \rightarrow V^{(2)}$ by

$$
x_{i}^{(1)} \xi= \begin{cases}x_{i}^{(2)} & \text { for } i \in\{1, \ldots, a(1)\} \\ x_{i}^{(2)}+w_{i}+x_{i \phi}^{(2)} & \text { for } i \in\{a(1)+1, \ldots, b(1)\} \\ w_{i}+x_{i \phi}^{(2)} & \text { for } i \in\{b(1)+1, \ldots, d(1)\} .\end{cases}
$$

Note that, in these equations, $x_{i}^{(2)} \in V_{1}^{(2)} \oplus \cdots \oplus V_{r(1)}^{(2)}$, while $w_{i} \in W$ and $x_{i \phi}^{(2)} \in$ $V_{r(2)}^{(2)} \oplus \cdots \oplus V_{m(2)+1}^{(2)}$, where $V_{1}^{(2)} \oplus \cdots \oplus V_{r(1)}^{(2)}, W$ and $V_{r(2)}^{(2)} \oplus \cdots \oplus V_{m(2)+1}^{(2)}$ are pairwise orthogonal. It is straightforward to check that $\theta^{(2)}\left(x_{i}^{(1)} \xi, x_{j}^{(1)} \xi\right)=\theta^{(1)}\left(x_{i}^{(1)}, x_{j}^{(1)}\right)$ in all the various cases for $i$ and $j$. Hence $\xi$ is a homomorphism of $S$-forms. Clearly $\xi$ has the form required in (3.1). Thus we have $Y^{(1)} \preccurlyeq Y^{(2)}$, as required.

PROOF OF THEOREM B. Take any positive integer $N$ such that $N \geq|K|\left(2|S|^{2}+|S|\right)$. Then, by Lemma 3.3, for each $S$-form $(V, \theta)$ there exists an ordered basis $X_{(V, \theta)}$ of $V$ such that $\left(V, \theta, X_{(V, \theta)}\right)$ is an $N$-regular $S$-triple. If $(V, \theta)$ and $\left(V^{\prime}, \theta^{\prime}\right)$ are $S$-forms such that $\left(V, \theta, X_{(V, \theta)}\right) \preccurlyeq\left(V^{\prime}, \theta^{\prime}, X_{\left(V^{\prime}, \theta^{\prime}\right)}\right)$ then $(V, \theta) \preccurlyeq\left(V^{\prime}, \theta^{\prime}\right)$. Hence the result follows by Proposition 3.5.

To prove our result about varieties of groups we need, in fact, not Theorem B itself but the assertion stated below as Proposition 3.7.

Let $T$ be any non-empty finite set. We consider finite sequences $\left(t_{1}, \ldots, t_{n}\right)$ of elements of $T$ and write $\left(t_{1}, \ldots, t_{n}\right) \preccurlyeq\left(t_{1}^{\prime}, \ldots, t_{n^{\prime}}^{\prime}\right)$ if $\left(t_{1}, \ldots, t_{n}\right)$ is a subsequence of $\left(t_{1}^{\prime}, \ldots, t_{n^{\prime}}^{\prime}\right)$, that is, if there is a one-one order-preserving map $\phi:\{1, \ldots, n\} \rightarrow$ $\left\{1, \ldots, n^{\prime}\right\}$ such that $t_{i}=t_{i \phi}^{\prime}$ for $i=1, \ldots, n$. Clearly $\preccurlyeq$ is a quasi-order (in fact a partial-order). The following result is a special case of [6, Theorem 4.3].

LEMMA 3.6. The set of all finite sequences of elements of $T$ is well-quasi-ordered under the relation $\preccurlyeq$. 
We define an $(S, T)$-form to be a quadruple $(V, \theta, X, \mathbf{t})$ where $(V, \theta, X)$ is an $S$ triple and $\mathbf{t}$ is an ordered $d$-tuple $\left(t_{1}, \ldots, t_{d}\right)$ of elements of $T$, with $d=\operatorname{rank}(V)$. We say that $(S, T)$-forms $(V, \theta, X, \mathbf{t})$ and $\left(V^{\prime}, \theta^{\prime}, X^{\prime}, \mathbf{t}^{\prime}\right)$ are isomorphic if the $S$-triples $(V, \theta, X)$ and $\left(V^{\prime}, \theta^{\prime}, X^{\prime}\right)$ are isomorphic and $\mathbf{t}=\mathbf{t}^{\prime}$. Let $\operatorname{rank}(V)=d, \operatorname{rank}\left(V^{\prime}\right)=d^{\prime}$, $X=\left\{x_{1}, \ldots, x_{d}\right\}$ and $X^{\prime}=\left\{x_{1}^{\prime}, \ldots, x_{d^{\prime}}^{\prime}\right\}$. Write $(V, \theta, X, \mathbf{t}) \preccurlyeq\left(V^{\prime}, \theta^{\prime}, X^{\prime}, \mathbf{t}^{\prime}\right)$ if there is a one-one order-preserving map $\phi:\{1, \ldots, d\} \rightarrow\left\{1, \ldots, d^{\prime}\right\}$ together with an $S$-form homomorphism $\xi:(V, \theta) \rightarrow\left(V^{\prime}, \theta^{\prime}\right)$ such that, for $i=1, \ldots, d, t_{i}=t_{i \phi}^{\prime}$ and

$$
x_{i} \xi=x_{i \phi}^{\prime}+z_{i}, \quad \text { for some } z_{i} \in\left\langle x_{1}^{\prime}, x_{2}^{\prime}, \ldots, x_{i \phi-1}^{\prime}\right\rangle .
$$

Clearly $\preccurlyeq$ is a quasi-order on the set of all $(S, T)$-forms, and we observe that $(V, \theta, X, \mathbf{t}) \preccurlyeq\left(V^{\prime}, \theta^{\prime}, X^{\prime}, \mathbf{t}^{\prime}\right)$ implies $(V, \theta, X) \preccurlyeq\left(V^{\prime}, \theta^{\prime}, X^{\prime}\right)$.

An $(S, T)$-form $(V, \theta, X, \mathbf{t})$ is said to be $N$-regular if the $S$-triple $(V, \theta, X)$ is $N$ regular. For given $S, T$ and $N$ we write $\mathscr{Z}$ for the set of all $N$-regular $(S, T)$-forms.

PROPOSITION 3.7. The set $(\mathscr{Z}, \preccurlyeq)$ is well-quasi-ordered.

PROOF. Let $Z^{(1)}, Z^{(2)}, Z^{(3)}, \ldots$ be an infinite sequence of $N$-regular $(S, T)$-forms. It suffices to show that there exist integers $i$ and $j$ with $i<j$ such that $Z^{(i)} \preccurlyeq Z^{(j)}$. For each $i$, let $Z^{(i)}=\left(V^{(i)}, \theta^{(i)}, X^{(i)}, \mathbf{t}^{(i)}\right)$ and use further notation for $\left(V^{(i)}, \theta^{(i)}, X^{(i)}\right)$ exactly as in the proof of Proposition 3.5. Also, write $\mathbf{t}^{(i)}=\left(t_{1}^{(i)}, \ldots, t_{d(i)}^{(i)}\right)$.

As in the proof of Proposition 3.5, we may assume that $\{m(1), m(2), \ldots\}$ is unbounded and we may pass to a subsequence with the property (3.2) for all $n \in \mathbb{N}$. But, for each $n$ and each $k \in\left\{1, \ldots, N^{[n]}\right\}$, there are only finitely many possibilities for $t_{k}^{(i)}$; thus we may also assume that, for all $k \in\left\{1, \ldots, N^{[n]}\right\}, t_{k}^{(i)}$ is independent of $i$ for all $i \geq n$.

Define $\bar{V}, \bar{X}, \bar{\theta}, P, Q$ and $r$ as before. Also, for each $k \in \mathbb{N}$, define $\bar{t}_{k}$ to be the limiting value of $t_{k}^{(i)}$. Then define $r(i)$ as before, but with the additional requirement that $t_{k}^{(i)}=\bar{t}_{k}$ for all $k \in\left\{1, \ldots, N^{[r(i)]}\right\}$.

Define $a(i)$ and $b(i)$ as before and pass to an infinite subsequence with property (3.3) for each $i$. Also, define $\mathbf{t}_{i}=\left(t_{a(i)+1}^{(i)}, t_{a(i)+2}^{(i)}, \ldots, t_{d(i)}^{(i)}\right)$ for each $i$. By Lemma 3.6, there exist $i$ and $j$ with $i<j$ such that $\mathbf{t}_{i}$ is a subsequence of $\mathbf{t}_{j}$. Hence, by passing to an infinite subsequence of $Z^{(1)}, Z^{(2)}, \ldots$, we may assume that $\mathbf{t}_{1}$ is a subsequence of $\mathbf{t}_{2}$. Thus there is a one-one order-preserving map $\phi:\{a(1)+1, \ldots, d(1)\} \rightarrow$ $\{a(2)+1, \ldots, d(2)\}$ such that $t_{i}^{(1)}=t_{i \phi}^{(2)}$ for $i=a(1)+1, \ldots, d(1)$. We may extend $\phi$ to a one-one order-preserving map $\phi:\{1, \ldots, d(1)\} \rightarrow\{1, \ldots, d(2)\}$ by defining $i \phi=i$ for $i=1, \ldots, a(1)$.

As in the proof of Proposition 3.5, there is a homomorphism of $S$-forms $\xi$ : $\left(V^{(1)}, \theta^{(1)}\right) \rightarrow\left(V^{(2)}, \theta^{(2)}\right)$ such that $\xi$ has the form required in (3.4). For $i=$ $1, \ldots, a(1)$, we have $t_{i}^{(1)}=t_{i}^{(2)}=\bar{t}_{i}$, since $a(1) \leq N^{[r(1)]} \leq N^{[r(2)]}$, and so $t_{i}^{(1)}=t_{i \phi}^{(2)}$, 
since $i=i \phi$. Also, for $i=a(1)+1, \ldots, d(1)$, we have $t_{i}^{(1)}=t_{i \phi}^{(2)}$ by the choice of $\phi$. Thus $Z^{(1)} \preccurlyeq Z^{(2)}$.

An alternating $S$-form is an $S$-form $(V, \theta)$ such that $\theta(v, v)=0$ for all $v \in V$. Consider now the case where $S=K$. An alternating $K$-form $(V, \theta)$ is called standard with respect to the ordered basis $\left\{x_{1}, \ldots, x_{d}\right\}$ of $V$ if $\theta\left(x_{i}, x_{j}\right)=0$ for all $i, j$ such that $1 \leq i<j \leq d$ and $(i, j) \notin\{(1,2),(3,4), \ldots,(2[d / 2]-1,2[d / 2])\}$.

LEMMA 3.8 (compare [2]). Let $n_{0}$ be an integer, with $n_{0} \geq 2$, and let $K=\mathbb{Z} / n_{0} \mathbb{Z}$. Let $(V, \theta)$ be an alternating $K$-form. Then there is a $K$-basis $\left\{x_{1}, \ldots, x_{d}\right\}$ of $V$ such that $(V, \theta)$ is standard with respect to $\left\{x_{1}, \ldots, x_{d}\right\}$.

PROOF. Choose $u_{1}, u_{2} \in V$ such that the additive cyclic subgroup $\left\langle\theta\left(u_{1}, u_{2}\right)\right\rangle$ of $K$ has largest possible order. Let $x_{1}$ be an element of $V$ of order $n_{0}$ such that $u_{1} \in\left\langle x_{1}\right\rangle$. Note that $x_{1}$ belongs to some basis of $V$. By maximality, $\left\langle\theta\left(u_{1}, u_{2}\right)\right\rangle=\left\langle\theta\left(x_{1}, u_{2}\right)\right\rangle$. Hence we may replace $u_{1}$ by $x_{1}$. Let $U$ be a submodule of $V$ such that $V=\left\langle x_{1}\right\rangle \oplus U$. If $U=\{0\}$ then $\left\{x_{1}\right\}$ is the required basis, so suppose $U \neq\{0\}$. Write $u_{2}=u_{2}^{\prime}+u$ where $u_{2}^{\prime} \in\left\langle x_{1}\right\rangle$ and $u \in U$. Clearly we may replace $u_{2}$ by $u$. Then, as before, we may replace $u$ by an element $x_{2}$ which belongs to a basis of $U$. Thus $\left\{x_{1}, x_{2}\right\}$ is contained in a basis of $V$. Set $W=\left\{w \in V: \theta\left(x_{1}, w\right)=\theta\left(x_{2}, w\right)=0\right\}$. Let $v \in V$. The choice of $x_{1}$ and $x_{2}$ shows that $\theta\left(x_{1}, x_{2}\right)$ is a generator of the cyclic group $\left\{\theta\left(x_{1}, u\right): u \in V\right\}$. Hence there exists $\lambda \in K$ such that $\theta\left(x_{1}, v\right)=\lambda \theta\left(x_{1}, x_{2}\right)$. Similarly there exists $\mu \in K$ such that $\theta\left(v, x_{2}\right)=\mu \theta\left(x_{1}, x_{2}\right)$. It follows that $v-\mu x_{1}-\lambda x_{2} \in W$ and so $v \in\left\langle x_{1}, x_{2}\right\rangle+W$. Therefore $V=\left\langle x_{1}, x_{2}\right\rangle+W$. Thus we may find a basis $\left\{x_{1}, x_{2}, w_{1}, \ldots, w_{d-2}\right\}$ of $V$ with $w_{1}, \ldots, w_{d-2} \in W$. The lemma follows by an inductive argument applied to $\left\langle w_{1}, \ldots, w_{d-2}\right\rangle$.

\section{Direct powers of finite groups}

In this section we shall obtain some results which will be useful for both Theorem C and Theorem D.

Let $G$ be a finite group and let $D$ be the (restricted) direct product $D=\prod_{i \in \mathrm{N}} G_{i}$ where $G_{i}=G$ for all $i$. Thus the elements of $D$ may be regarded as sequences of the form $\left(g_{1}, g_{2}, \ldots\right)$ where $g_{i} \in G$ for all $i$ and where $\left\{i: g_{i} \neq 1\right\}$ is finite.

Let $\phi: \mathbb{N} \rightarrow \mathbb{N}$ be a one-one order-preserving function. Let $X$ be a finite subset of $\mathbb{N} \backslash \mathbb{N} \phi$ and let $\sigma: X \rightarrow \mathbb{N} \phi$ be a function such that $j<j \sigma$ for all $j \in X$. Given such $\phi, X$ and $\sigma$, let $\xi$ be the endomorphism of $D$ defined by

$$
\left(g_{1}, g_{2}, \ldots\right) \xi=\left(g_{1}^{\prime}, g_{2}^{\prime}, \ldots\right)
$$


where $g_{j}^{\prime}=g_{i}$ if $j=i \phi, g_{j}^{\prime}=1$ if $j \notin \mathbb{N} \phi \cup X$, and $g_{j}^{\prime}=g_{j \sigma}^{\prime}$ if $j \in X$. Let $\Xi$ be the set of all such endomorphisms of $D$ (for all possible choices of $\phi, X$ and $\sigma$ ).

Let $\leq$ be a total order on $G$ which is arbitrary except that $1 \leq g$ for all $g \in G$. Then the set $D$ may be ordered lexicographically from the right: if $d, d^{\prime} \in D$ where $d=\left(g_{1}, g_{2}, \ldots\right)$ and $d^{\prime}=\left(g_{1}^{\prime}, g_{2}^{\prime}, \ldots\right)$, we set $d<d^{\prime}$ if there exists $l \in \mathbb{N}$ such that $g_{l}<g_{l}^{\prime}$ but $g_{i}=g_{i}^{\prime}$ for all $i>l$. Clearly $(D, \leq)$ is well-ordered, and it is easy to prove the following result.

Lemma 4.1. Let $d, d^{\prime} \in D$ and let $\xi \in \Xi$. If $d<d^{\prime}$ then $d \xi<d^{\prime} \xi$.

For $d \in D$, where $d=\left(g_{1}, g_{2}, \ldots\right)$, write

$$
\operatorname{span}(d)=\left\{g \in G \backslash\{1\}: g=g_{i} \text { for some } i\right\},
$$

and, for $g \in \operatorname{span}(d)$, let $i_{g}(d)$ denote the largest $i$ such that $g_{i}=g$.

Let $d$ and $d^{\prime}$ be elements of $D$, where $d=\left(g_{1}, g_{2}, \ldots\right)$ and $d^{\prime}=\left(g_{1}^{\prime}, g_{2}^{\prime}, \ldots\right)$. Write $d \preccurlyeq d^{\prime}$ if $\operatorname{span}(d)=\operatorname{span}\left(d^{\prime}\right)$ and there is a one-one order-preserving function $\phi: \mathbb{N} \rightarrow \mathbb{N}$ such that $g_{i}=g_{i \phi}^{\prime}$ for all $i$ and $i_{g}(d) \phi=i_{g}\left(d^{\prime}\right)$ for all $g \in \operatorname{span}(d)$. Clearly $(D, \preccurlyeq)$ is quasi-ordered (in fact, partially-ordered).

LEMMA 4.2. The set $(D, \preccurlyeq)$ is well-quasi-ordered.

ProOF. Let $m=|G \backslash\{1\}|$ and assume $m \geq 1$ (the result is trivial for $m=0$ ). Write $G \backslash\{1\}=\left\{a_{1}, \ldots, a_{m}\right\}$. For $d \in D$ and $k=1, \ldots, m$, define $p_{k}(d)=i_{a_{k}}(d)$ if $a_{k} \in \operatorname{span}(d)$ and $p_{k}(d)=1$ otherwise, so that we obtain an $m+1$-tuple $s(d)=$ $\left(p_{1}(d), \ldots, p_{m}(d), d\right)$. Let $d, d^{\prime} \in D$, where $d=\left(g_{1}, g_{2}, \ldots\right)$ and $d^{\prime}=\left(g_{1}^{\prime}, g_{2}^{\prime}, \ldots\right)$. Following the notation of [3], we write $s(d) \preccurlyeq_{\Phi} s\left(d^{\prime}\right)$ if there exists a one-one orderpreserving map $\phi: \mathbb{N} \rightarrow \mathbb{N}$ such that $g_{i}=g_{i \phi}^{\prime}$ for all $i$ and $p_{i}(d) \phi=p_{i}\left(d^{\prime}\right)$ for $i=1, \ldots, m$. By [3, Lemma 3.2], the set of $m+1$-tuples $s(d)$ is well-quasi-ordered under $\preccurlyeq_{\Phi}$. But $s(d) \preccurlyeq_{\Phi} s\left(d^{\prime}\right)$ implies $d \preccurlyeq d^{\prime}$. The result follows.

Let $\mathbb{F}$ be any field. Then each non-zero element $u$ of the group algebra $\mathbb{F} D$ can be written (uniquely) in the form $u=\lambda_{1} d_{1}+\cdots+\lambda_{r} d_{r}$ where $d_{1}, \ldots, d_{r} \in D$, $d_{1}>\cdots>d_{r}$ and $\lambda_{1}, \ldots, \lambda_{r} \in \mathbb{F} \backslash\{0\}$. The largest group element $d_{1}$ is called the leading group element of $u$ and we write $d_{1}=\operatorname{lead}(u)$. Since every endomorphism of $D$ extends to $\mathbb{F} D$, each element of $\Xi$ acts on $\mathbb{F} D$. For $S \subseteq \mathbb{F} D$ we write $\langle S\rangle_{\Xi}$ for the $\Xi$-closed subspace of $\mathbb{F} D$ generated by $S$.

LEMMA 4.3. Let $u$ and $v$ be non-zero elements of $\mathbb{F} D$ with $\operatorname{lead}(u) \preccurlyeq \operatorname{lead}(v)$. Then there exists $v^{*} \in \mathbb{F} D$ such that $\langle u, v\rangle_{\Xi}=\left\langle u, v^{*}\right\rangle_{\Xi}$ and either $v^{*}=0$ or $\operatorname{lead}\left(v^{*}\right)<\operatorname{lead}(v)$. 
PROOF. Write $u=\lambda_{1} d_{1}+\cdots+\lambda_{r} d_{r}$ and $v=\lambda_{1}^{\prime} d_{1}^{\prime}+\cdots+\lambda_{s}^{\prime} d_{s}^{\prime}$ where the $d_{i}$ and $d_{i}^{\prime}$ are elements of $D, d_{1}>\cdots>d_{r}, d_{1}^{\prime}>\cdots>d_{s}^{\prime}$, and the $\lambda_{i}$ and $\lambda_{i}^{\prime}$ are elements of $\mathbb{F} \backslash\{0\}$. Write $d=d_{1}=\operatorname{lead}(u)$ and $d^{\prime}=d_{1}^{\prime}=\operatorname{lead}(v)$. Thus $d \preccurlyeq d^{\prime}$. Let $d=\left(g_{1}, g_{2}, \ldots\right)$ and $d^{\prime}=\left(g_{1}^{\prime}, g_{2}^{\prime}, \ldots\right)$, and let $\phi: \mathbb{N} \rightarrow \mathbb{N}$ be as in the definition of $d \preccurlyeq d^{\prime}$. Let $X=\left\{j: j \notin \mathbb{N} \phi\right.$ and $\left.g_{j}^{\prime} \neq 1\right\}$. By the definition of $d \preccurlyeq d^{\prime}$ we have $i_{g}\left(d^{\prime}\right) \in \mathbb{N} \phi$ for all $g \in \operatorname{span}\left(d^{\prime}\right)$. For each $j \in X$ let $j \sigma=i_{g}\left(d^{\prime}\right)$ where $g=g_{j}^{\prime}$. Let $\xi$ be the element of $\Xi$ corresponding to $\phi, X$ and $\sigma$. Then it is easy to check that $d \xi=d^{\prime}$. Hence, by Lemma 4.1, $\operatorname{lead}(u \xi)=d^{\prime}=\operatorname{lead}\left(v^{\prime}\right)$. Let $v^{*}=v-\lambda_{1}^{\prime} \lambda_{1}^{-1}(u \xi)$. Then the result follows.

PROPOSITION 4.4. The maximal condition holds for $\Xi$-closed subspaces of $\mathbb{F} D$.

PROOF. Let $U$ be a $\Xi$-closed subspace of $F D$. It suffices to prove that $U$ is finitely generated as a $\Xi$-closed subspace. By Lemma 4.2, there exists a finite subset $S$ of $U \backslash\{0\}$ such that for all $v \in U \backslash\{0\}$ there exists $u \in S$ such that lead $(u) \preccurlyeq \operatorname{lead}(v)$. We claim that $U=\langle S\rangle_{\Xi}$. Suppose, in order to get a contradiction, that there exists $v \in U$ such that $v \notin\langle S\rangle_{\Xi}$, and choose such $v$ so that lead $(v)$ is as small as possible in the well-ordered set $(D, \leq)$. There exists $u \in S$ such that lead $(u) \preccurlyeq \operatorname{lead}(v)$. By Lemma 4.3, there exists $v^{*} \in \mathbb{F} D$ such that $\langle u, v\rangle_{\Xi}=\left\langle u, v^{*}\right\rangle_{\Xi}$ and either $v^{*}=0$ or lead $\left(v^{*}\right)<\operatorname{lead}(v)$. Since $v \notin\langle u\rangle_{\Xi}$, we have $v^{*} \neq 0$. Since $v^{*} \in\langle u, v\rangle_{\Xi} \subseteq U$, the choice of $v$ gives $v^{*} \in\langle S\rangle_{\Xi}$. Hence $v \in\left\langle u, v^{*}\right\rangle_{\Xi} \subseteq\langle S\rangle_{\Xi}$, and we have the required contradiction.

Let $n$ be a positive integer and let $E$ be a free group of countably infinite rank in the variety $\mathbf{A}_{n}$. Let $\Gamma$ be the set of all endomorphisms of $E$.

PROPOSITION 4.5. For each positive integer $r$, the maximal condition holds for $\Gamma$-closed subspaces of $\mathbb{F}\left(E^{\times r}\right)$.

PROOF. Clearly we may assume $n>1$. Let $\left\{x_{1}, x_{2}, \ldots\right\}$ be a free generating set for $E$. For each $i \in \mathbb{N}$, let $G_{i}$ be the subgroup of $E^{\times r}$ generated by the elements $\left(x_{i}, 1, \ldots, 1\right),\left(1, x_{i}, 1, \ldots, 1\right), \ldots,\left(1, \ldots, 1, x_{i}\right)$. Write $G=G_{1}$. Thus $G$ is a finite group. Clearly $E^{\times r}$ is the direct product of the groups $G_{i}$ and, for each $i$, there is an obvious isomorphism from $G$ to $G_{i}$. Thus we may identify $E^{\times r}$ with the direct power $D$ of $G$ considered above. The result will follow from Proposition 4.4 if we can show that every element of $\Xi$ is induced by some element of $\Gamma$. Let $\xi \in \Xi$ and suppose that $\xi$ is associated with $\phi, X$ and $\sigma$, in the notation used before. Define a homomorphism $\gamma: E \rightarrow E$ by $x_{i} \gamma=x_{i \phi} \prod_{j \in X, j \sigma=i \phi} x_{j}$, for each $i$, where the product is taken over all those values of $j$, if any, which lie in $X$ and satisfy $j \sigma=i \phi$. It is straightforward to verify that $\gamma$ induces $\xi$. 


\section{Proof of Theorem $\mathrm{C}$}

We use the notation of Section 1. In particular, $n$ is a positive integer, $A$ is a free group of $\mathbf{N}_{2, n}$ of countably infinite rank, $\Psi$ is the set of endomorphisms of $A$ and $\mathbb{F}$ is a field of characteristic not dividing $n$. We shall describe the proof of Theorem $\mathrm{C}$ only in the case $r=2$. The proof for general $r$ is essentially the same, but greater notational complexity is required for $r>2$.

Let $\overline{\mathbb{F}}$ be the algebraic closure of $\mathbb{F}$. If $I$ is a $\Psi$-closed left ideal of $\mathbb{F}(A \times A)$ then $\overline{\mathbb{F}} \otimes_{\mathbb{F}} I$ is a $\Psi$-closed left ideal of $\overline{\mathbb{F}}(A \times A)$, and $I=\mathbb{F}(A \times A) \cap \overline{\mathbb{F}} \otimes_{\mathbb{F}} I$. Therefore we may assume that $\mathbb{F}=\overline{\mathbb{F}}$. We write $\mathbb{F}^{\times}$for the multiplicative group $\mathbb{F} \backslash\{0\}$.

Let $\left\{x_{i}: i \in \mathbb{N}\right\}$ be a free generating set of $A$ and, for each positive integer $k$, let $A_{k}$ be the subgroup $\left\langle x_{1}, \ldots, x_{k}\right\rangle$. Define $n_{0}$ by $n_{0}=n$ if $n$ is odd and $n_{0}=n / 2$ if $n$ is even. For all $a, b \in A$ we have $(a b)^{n}=1$ and hence $[a, b]^{n_{0}}=\left[a^{n_{0}}, b\right]=1$. Thus $\left(A^{\prime}\right)^{n_{0}}=\{1\}$ and $A^{n_{0}}$ is central in $A$. It is easily verified that the relations $x_{i}^{n}=1$ and $\left[x_{i}, x_{j}\right]^{n_{0}}=1$, for all $i, j \in\{1, \ldots, k\}$, imposed on the free nilpotent group of class 2 on free generators $x_{1}, \ldots, x_{k}$, give a group of exponent $n$, which is therefore isomorphic to $A_{k}$. It follows that $A_{k}^{\prime}$ is a free abelian group of exponent $n_{0}$ with basis $\left\{\left[x_{i}, x_{j}\right]: 1 \leq i<j \leq k\right\}$. If $n \leq 2$, then $A$ is the free group of countably infinite rank in the variety $\mathbf{A}_{n}$, and, in this case, Theorem $C$ follows from Proposition 4.5. Thus we assume that $n>2$, so that $n_{0}>1$.

Let $K=\mathbb{Z} / n_{0} \mathbb{Z}$ and let $\omega$ be a primitive $n_{0}$-th root of unity in $\mathbb{F}$. Thus $\omega^{\lambda}$ is well-defined for all $\lambda \in K$, and $\left\{\omega^{\lambda}: \lambda \in K\right\}$ is the cyclic subgroup of $\mathbb{F}^{\times}$consisting of all $n_{0}$-th roots of unity in $\mathbb{F}$.

Let $Q_{k}$ be the set of all ordered pairs $(i, j)$ with $1 \leq i<j \leq k$, and let $\Delta_{k}$ be the set of all functions $\delta: Q_{k} \rightarrow K$. For each $\delta \in \Delta_{k}$ there is a group homomorphism $\chi_{\delta}: A_{k}^{\prime} \rightarrow \mathbb{F}^{\times}$determined by $\chi_{\delta}\left(\left[x_{i}, x_{j}\right]\right)=\omega^{\delta(i, j)}$ for all $(i, j) \in Q_{k}$. Since the elements $\left[x_{i}, x_{j}\right]$ form a basis for $A_{k}^{\prime}$, every homomorphism $A_{k}^{\prime} \rightarrow \mathbb{F}^{\times}$arises in this way from some $\delta$. We extend $\chi_{\delta}$ by linearity to a function $\chi_{\delta}: \mathbb{F} A_{k}^{\prime} \rightarrow \mathbb{F}$. In the language of representation theory, the functions $\chi_{\delta}$ are the characters afforded by the irreducible representations of the abelian group $A_{k}^{\prime}$ over $\mathbb{F}$, all of which are one-dimensional.

For each $\delta \in \Delta_{k}$, let $e_{\delta}$ be the element of $\mathbb{F} A_{k}^{\prime}$ defined by

$$
e_{\delta}=\frac{1}{\left|A_{k}^{\prime}\right|} \sum_{a \in A_{k}^{\prime}} \chi_{\delta}\left(a^{-1}\right) a .
$$

The elements $e_{\delta}$ have the following properties, which may be verified by elementary representation theory or direct calculation.

$$
\begin{gathered}
w e_{\delta}=\chi_{\delta}(w) e_{\delta} \text { for all } \delta \in \Delta_{k} \text { and all } w \in \mathbb{F} A_{k}^{\prime} . \\
\chi_{\delta}\left(e_{\delta}\right)=1 \text { and } e_{\delta}^{2}=e_{\delta} \text { for all } \delta \in \Delta_{k} .
\end{gathered}
$$




$$
\chi_{\delta^{\prime}}\left(e_{\delta}\right)=0 \text { and } e_{\delta} e_{\delta^{\prime}}=0 \text { for all } \delta, \delta^{\prime} \in \Delta_{k} \text { with } \delta \neq \delta^{\prime} \text {. }
$$

$$
\sum_{\delta \in \Delta_{k}} e_{\delta}=1
$$

Thus the elements $e_{\delta}$ are pairwise orthogonal idempotents. They form a basis of $\mathbb{F} A_{k}^{\prime}$ and each $e_{\delta}$ spans a one-dimensional ideal of $\mathbb{F} A_{k}^{\prime}$. Within the larger group algebras $\mathbb{F} A_{k}$ and $\mathbb{F} A$, the $e_{\delta}$ are central idempotents. For each $\delta$, let $I_{\delta}=\left(\mathbb{F} A_{k}\right) e_{\delta}$. Thus $I_{\delta}$ is the (two-sided) ideal of $\mathbb{F} A_{k}$ generated by $e_{\delta}$. By (5.3), (5.4) and (5.5),

$$
\mathbb{F} A_{k}=\bigoplus_{\delta \in \Delta_{k}} I_{\delta}
$$

It follows from (5.6) and (5.2) that $\mathbb{F} A_{k}$ is spanned by all elements of the form $x_{1}^{\alpha_{1}} \cdots x_{k}^{\alpha_{k}} e_{\delta}$ with $\delta \in \Delta_{k}$ and $\alpha_{i} \in \mathbb{Z} / n \mathbb{Z}$ for $i=1, \ldots, k$. It is easily checked that there are exactly $\left|A_{k}\right|$ such elements. Hence they form a basis for $\mathbb{F} A_{k}$ and, for fixed $\delta$, the elements $x_{1}^{\alpha_{1}} \cdots x_{k}^{\alpha_{k}} e_{\delta}$ form a basis for $I_{\delta}$.

If $\psi: A_{k} \rightarrow A_{l}$ is a homomorphism, where $k, l \in \mathbb{N}$, then $\psi$ extends to a homomorphism $\mathbb{F} A_{k} \rightarrow \mathbb{F} A_{l}$, which we also denote by $\psi$. In particular, $\psi: A_{k} \rightarrow A_{k}$ extends to $\psi: \mathbb{F} A_{k} \rightarrow \mathbb{F} A_{k}$.

For each $k$, write $\tilde{A}_{k}=A_{k} / A_{k}^{\prime}\left(A_{k}\right)^{n_{0}}$ and, for $a \in A_{k}$, write $\tilde{a}=a A_{k}^{\prime}\left(A_{k}\right)^{n_{0}} \in \tilde{A}_{k}$. Thus $\tilde{A}_{k}$ is a free abelian group of exponent $n_{0}$ with basis $\left\{\tilde{x}_{1}, \ldots, \tilde{x}_{k}\right\}$. We shall usually think of $\tilde{A}_{k}$ in additive notation: thus we may regard it as a free $K$-module.

If $\psi: A_{k} \rightarrow A_{l}$ is a homomorphism, we write $\tilde{\psi}$ for the induced homomorphism from $\tilde{A}_{k}$ to $\tilde{A}_{l}$. In particular, if $\eta \in \operatorname{Aut}\left(A_{k}\right)$ then $\tilde{\eta} \in \operatorname{Aut}\left(\tilde{A}_{k}\right)$.

For each $\delta \in \Delta_{k}$, let $\theta_{\delta}$ be the alternating $K$-form on $\tilde{A}_{k}$ satisfying $\theta_{\delta}\left(\tilde{x}_{i}, \tilde{x}_{j}\right)=$ $\delta(i, j)$ for all $(i, j) \in Q_{k}$. Clearly every alternating $K$-form on $\tilde{A}_{k}$ arises in this way from some $\delta$. Since $\chi_{\delta}\left(\left[x_{i}, x_{j}\right]\right)=\omega^{\delta(i, j)}$ it is straightforward to verify that

$$
\chi_{\delta}\left(\left[a_{1}, a_{2}\right]\right)=\omega^{\theta_{\delta}\left(\tilde{a}_{1}, \tilde{a}_{2}\right)} \text { for all } a_{1}, a_{2} \in A_{k} .
$$

Lemma 5.1. Let $\delta \in \Delta_{k}$ and $\eta \in \operatorname{Aut}\left(A_{k}\right)$. Then $e_{\delta} \eta=e_{\varepsilon}$ where $\varepsilon \in \Delta_{k}$ and $\theta_{\varepsilon}\left(\tilde{a}_{1}, \tilde{a}_{2}\right)=\theta_{\delta}\left(\tilde{a}_{1} \tilde{\eta}^{-1}, \tilde{a}_{2} \tilde{\eta}^{-1}\right)$ for all $a_{1}, a_{2} \in A_{k}$.

PROOF. The map $a \mapsto \chi_{\delta}\left(a \eta^{-1}\right)$ is a homomorphism from $A_{k}^{\prime}$ to $\mathbb{F}^{x}$. Hence there exists $\varepsilon \in \Delta_{k}$ such that $\chi_{\varepsilon}(a)=\chi_{\delta}\left(a \eta^{-1}\right)$ for all $a \in A_{k}^{\prime}$. By direct calculation we obtain $e_{\delta} \eta=e_{\varepsilon}$. Also, for all $a_{1}, a_{2} \in A_{k}$, (5.7) gives $\omega^{\theta_{\varepsilon}\left(\bar{a}_{1}, \tilde{a}_{2}\right)}=\chi_{\varepsilon}\left(\left[a_{1}, a_{2}\right]\right)=$ $\chi_{\delta}\left(\left[a_{1}, a_{2}\right] \eta^{-1}\right)=\chi_{\delta}\left(\left[a_{1} \eta^{-1}, a_{2} \eta^{-1}\right]\right)=\omega^{\theta_{s}\left(\tilde{a}_{1} \tilde{\eta}^{-1}, \tilde{a}_{2} \tilde{\eta}^{-1}\right)}$. The result follows.

LEMMA 5.2. Let $\delta \in \Delta_{k}$ and $\varepsilon \in \Delta_{l}$, where $k, l \in \mathbb{N}$. Let $\psi: A_{k} \rightarrow A_{l}$ be a homomorphism which induces a homomorphism of $K$-forms from $\left(\tilde{A}_{k}, \theta_{\delta}\right)$ to $\left(\tilde{A}_{l}, \theta_{\varepsilon}\right)$ (that is, $\theta_{\delta}\left(\tilde{a}_{1}, \tilde{a}_{2}\right)=\theta_{\varepsilon}\left(\tilde{a}_{1} \tilde{\psi}, \tilde{a}_{2} \tilde{\psi}\right)$ for all $\left.a_{1}, a_{2} \in A_{k}\right)$. Then $\left(e_{\delta} \psi\right) e_{\varepsilon}=e_{\varepsilon}$. 
PROOF. For all $a_{1}, a_{2} \in A_{k}$,

$$
\chi_{\delta}\left(\left[a_{1}, a_{2}\right]\right)=\omega^{\theta_{\delta}\left(\tilde{a}_{1}, \tilde{a}_{2}\right)}=\omega^{\theta_{\varepsilon}\left(\tilde{a}_{1} \tilde{\psi}, \tilde{a}_{2} \tilde{\psi}\right)}=\chi_{\varepsilon}\left(\left[a_{1} \psi, a_{2} \psi\right]\right)=\chi_{\varepsilon}\left(\left[a_{1}, a_{2}\right] \psi\right) .
$$

It follows that $\chi_{\delta}(w)=\chi_{\varepsilon}(w \psi)$ for all $w \in \mathbb{F} A_{k}^{\prime}$. Therefore, by (5.2) and (5.3),

$$
\left(e_{\delta} \psi\right) e_{\varepsilon}=\chi_{\varepsilon}\left(e_{\delta} \psi\right) e_{\varepsilon}=\chi_{\delta}\left(e_{\delta}\right) e_{\varepsilon}=e_{\varepsilon} .
$$

For each $k$, we consider $\mathbb{F}\left(A_{k} \times A_{k}\right)$, identified with $\mathbb{F} A_{k} \otimes_{\mathbb{F}} \mathbb{F} A_{k}$. If $\psi: A_{k} \rightarrow A_{l}$ is a homomorphism, then $\psi$ yields homomorphisms $\psi: A_{k} \times A_{k} \rightarrow A_{l} \times A_{l}$ and $\psi: \mathbb{F}\left(A_{k} \times A_{k}\right) \rightarrow \mathbb{F}\left(A_{l} \times A_{l}\right)$. For $\delta, \delta^{\prime} \in \Delta_{k}$, we write $e_{\delta} \otimes e_{\delta^{\prime}}$ as $e_{\delta \delta^{\prime}}$ and $I_{\delta} \otimes I_{\delta^{\prime}}$ as $I_{\delta \delta^{\prime}}$. Thus, by (5.6),

$$
\mathbb{F}\left(A_{k} \times A_{k}\right)=\bigoplus_{\delta, \delta^{\prime} \in \Delta_{k}} I_{\delta \delta^{\prime}}
$$

Also, $I_{\delta \delta^{\prime}}$ is the ideal of $\mathbb{F}\left(A_{k} \times A_{k}\right)$ generated by the central idempotent $e_{\delta \delta^{\prime}}$, and $\sum_{\delta, \delta^{\prime}} e_{\delta \delta^{\prime}}=1$.

For $\delta, \delta^{\prime} \in \Delta_{k}$, let $\theta_{\delta \delta^{\prime}}$ be the alternating $K \oplus K$-form on $\tilde{A}_{k}$ determined by $\theta_{\delta \delta^{\prime}}\left(\tilde{x}_{i}, \tilde{x}_{j}\right)=\left(\theta_{\delta}\left(\tilde{x}_{i}, \tilde{x}_{j}\right), \theta_{\delta^{\prime}}\left(\tilde{x}_{i}, \tilde{x}_{j}\right)\right)$ for all $(i, j) \in Q_{k}$. Every alternating $K \oplus K$ form on $\tilde{A}_{k}$ arises in this way from some $\delta, \delta^{\prime}$.

The following two results are easily deduced from Lemma 5.1 and Lemma 5.2, respectively.

LEMMA 5.3. Let $\delta, \delta^{\prime} \in \Delta_{k}$ and $\eta \in \operatorname{Aut}\left(A_{k}\right)$. Then $e_{\delta \delta^{\prime}} \eta=e_{\varepsilon \varepsilon^{\prime}}$ where $\varepsilon, \varepsilon^{\prime} \in \Delta_{k}$ and $\theta_{\varepsilon \varepsilon^{\prime}}\left(\tilde{a}_{1}, \tilde{a}_{2}\right)=\theta_{\delta \delta^{\prime}}\left(\tilde{a}_{1} \tilde{\eta}^{-1}, \tilde{a}_{2} \tilde{\eta}^{-1}\right)$ for all $a_{1}, a_{2} \in A_{k}$.

LEMMA 5.4. Let $\delta, \delta^{\prime} \in \Delta_{k}$ and $\varepsilon, \varepsilon^{\prime} \in \Delta_{l}$, where $k, l \in \mathbb{N}$. Let $\psi: A_{k} \rightarrow A_{l}$ be a homomorphism which induces a homomorphism of $K \oplus K$-forms from $\left(\tilde{A}_{k}, \theta_{\delta \delta^{\prime}}\right)$ to $\left(\tilde{A}_{l}, \theta_{\varepsilon \varepsilon^{\prime}}\right)$. Then $\left(e_{\delta \delta^{\prime}} \psi\right) e_{\varepsilon \varepsilon^{\prime}}=e_{\varepsilon \varepsilon^{\prime}}$.

Let $N=n_{0}\left(2 n_{0}^{4}+n_{0}^{2}\right)$. By Lemma 3.3, every $K \oplus K$-form is $N$-regular with respect to some basis. For $\delta, \delta^{\prime} \in \Delta_{k}$, we say that $\theta_{\delta \delta^{\prime}}$ is regular if it is $N$-regular with respect to the basis $\left\{\tilde{x}_{1}, \ldots, \tilde{x}_{k}\right\}$ of $\tilde{A}_{k}$.

Lemma 5.5. Let $\delta, \delta^{\prime} \in \Delta_{k}$. Then there exists $\eta \in \operatorname{Aut}\left(A_{k}\right)$ such that $e_{\delta \delta^{\prime}} \eta=e_{\varepsilon \varepsilon^{\prime}}$ where $\varepsilon, \varepsilon^{\prime} \in \Delta_{k}$ and $\theta_{\varepsilon \varepsilon^{\prime}}$ is regular.

PROOF. By Lemma 3.3, there is a basis $\left\{\tilde{a}_{1}, \ldots, \tilde{a}_{k}\right\}$ of $\tilde{A}_{k}$ such that $\left(\tilde{A}_{k}, \theta_{\delta \delta^{\prime}}\right)$ is $N$-regular with respect to this basis. It is easily verified that there exists a generating set $\left\{y_{1}, \ldots, y_{k}\right\}$ of $A_{k}$ such that $\tilde{y}_{i}=\tilde{a}_{i}$ for $i=1, \ldots, k$. Since $A_{k}$ is a finite relatively free group of rank $k$, it follows that $\left\{y_{1}, \ldots, y_{k}\right\}$ is a free generating set. Let $\eta$ be the automorphism of $A_{k}$ satisfying $y_{i} \eta=x_{i}$ for $i=1, \ldots, k$. By Lemma 5.3, $e_{\delta \delta^{\prime}} \eta=e_{\varepsilon \varepsilon^{\prime}}$ where $\theta_{\varepsilon \varepsilon^{\prime}}\left(\tilde{x}_{i}, \tilde{x}_{j}\right)=\theta_{\delta \delta^{\prime}}\left(\tilde{y}_{i}, \tilde{y}_{j}\right)$ for all $(i, j) \in Q_{k}$. Thus $\theta_{\varepsilon \varepsilon^{\prime}}$ is regular. 
LEMMA 5.6. Let $\delta, \delta^{\prime} \in \Delta_{k}$ and $\varepsilon, \varepsilon^{\prime} \in \Delta_{l}$, where $k, l \in \mathbb{N}$, and consider $I_{\delta \delta^{\prime}}$ and $I_{\varepsilon \varepsilon^{\prime}}$ as subsets of $\mathbb{F}(A \times A)$. Then $I_{\delta \delta^{\prime}} \cap I_{\varepsilon \varepsilon^{\prime}}=\{0\}$ unless $k=l, \delta=\delta^{\prime}$ and $\varepsilon=\varepsilon^{\prime}$.

PROOF. Suppose that $k<l$. It is easily verified that $\left(\left[x_{l-1}, x_{l}\right] \otimes 1\right) w \notin \mathbb{F}\left(A_{k} \times A_{k}\right)$ for all $w \in \mathbb{F}\left(A_{k} \times A_{k}\right) \backslash\{0\}$. On the other hand, for all $v \in I_{\varepsilon \varepsilon^{\prime}}$, the element $\left(\left[x_{l-1}, x_{l}\right] \otimes 1\right) v$ is a scalar multiple of $v$ by (5.2). Thus $I_{\delta \delta^{\prime}} \cap I_{\varepsilon \varepsilon^{\prime}}=\{0\}$. If $k=l$ then $I_{\delta \delta^{\prime}} \cap I_{\varepsilon \varepsilon^{\prime}} \neq\{0\}$ implies $\delta=\delta^{\prime}$ and $\varepsilon=\varepsilon^{\prime}$ by (5.8).

A non-zero element $w$ of $\mathbb{F}(A \times A)$ will be called regular if $w \in I_{\delta \delta^{\prime}}$ for some $k$ and some $\delta, \delta^{\prime} \in \Delta_{k}$ such that $\theta_{\delta \delta^{\prime}}$ is regular. (By Lemma 5.6, $k, \delta$ and $\delta^{\prime}$ are then unique.)

LEMMA 5.7. Every $\Psi$-closed left ideal of $\mathbb{F}(A \times A)$ is generated, as a $\Psi$-closed vector space, by regular elements.

ProOF. Let $J$ be a $\Psi$-closed left ideal of $\mathbb{F}(A \times A)$ and let $J_{0}$ be the vector space spanned by all elements $v \psi$ where $v$ is a regular element of $J$ and $\psi \in \Psi$. It suffices to show that $J=J_{0}$. Clearly $J_{0} \subseteq J$. Let $w \in J$. Then $w \in \mathbb{F}\left(A_{k} \times A_{k}\right)$ for some $k$, and we have $w=\left(\sum_{\delta, \delta^{\prime} \in \Delta_{k}} e_{\delta \delta^{\prime}}\right) w=\sum_{\delta, \delta^{\prime} \in \Delta_{k}}\left(e_{\delta \delta^{\prime}} w\right)$, where $e_{\delta \delta^{\prime}} w \in J \cap I_{\delta \delta^{\prime}}$. It suffices to show that $e_{\delta \delta^{\prime}} w \in J_{0}$. Clearly we may assume that $e_{\delta \delta^{\prime}} w \neq 0$. By Lemma 5.5, there exists $\eta \in \operatorname{Aut}\left(A_{k}\right)$ such that $\left(e_{\delta \delta^{\prime}} w\right) \eta$ is regular. But $\left(e_{\delta \delta^{\prime}} w\right) \eta \in J$, since $\eta$ extends to an automorphism of $A$. Thus $e_{\delta \delta^{\prime}} w=\left(e_{\delta \delta^{\prime}} w\right) \eta \eta^{-1} \in J_{0}$.

Let $\delta, \delta^{\prime} \in \Delta_{k}$. Since the elements $x_{1}^{\alpha_{1}} \cdots x_{k}^{\alpha_{k}} e_{\delta}$ with $\alpha_{i} \in \mathbb{Z} / n \mathbb{Z}$ form a basis of $I_{\delta}$, the elements

$$
\left(x_{1}^{\alpha_{1}} \cdots x_{k}^{\alpha_{k}} \otimes x_{1}^{\alpha_{1}^{\prime}} \cdots x_{k}^{\alpha_{k}^{\prime}}\right) e_{\delta \delta^{\prime}},
$$

with $\alpha_{i}, \alpha_{i}^{\prime} \in \mathbb{Z} / n \mathbb{Z}$, form a basis of $I_{\delta \delta^{\prime}}$.

An element of $\mathbb{F}(A \times A)$ will be called a monomial if it has the form (5.9) for some $k$ and some $\delta, \delta^{\prime} \in \Delta_{k}$, and a regular monomial if $\theta_{\delta \delta^{\prime}}$ is regular. We write $\mathscr{M}$ for the set of all monomials, $\mathscr{M}^{*}$ for the set of all regular monomials, and $\mathscr{M}_{\delta \delta^{\prime}}$ for the set of all monomials of $I_{\delta \delta^{\prime}}$.

Let $T=\mathbb{Z} / n \mathbb{Z} \times \mathbb{Z} / n \mathbb{Z}$, that is, the Cartesian square of the set $\mathbb{Z} / n \mathbb{Z}$. With the monomial (5.9) we associate the $k$-tuple $\left(t_{1}, t_{2}, \ldots, t_{k}\right)$ where $t_{i}=\left(\alpha_{i}, \alpha_{i}^{\prime}\right) \in T$ for $i=1, \ldots, k$. Let $\leq$ be a total order on $T$ which is arbitrary except that $(0,0) \leq t$ for all $t \in T$. Then the set of all $k$-tuples of elements of $T$ can be ordered lexicographically from the right: if $\mathbf{t}=\left(t_{1}, \ldots, t_{k}\right)$ and $\mathbf{t}^{\prime}=\left(t_{1}^{\prime}, \ldots, t_{k}^{\prime}\right)$ are two such $k$-tuples, we set $\mathbf{t}<\mathbf{t}^{\prime}$ if there exists $q \in\{1, \ldots, k\}$ such that $t_{q}<t_{q}^{\prime}$ but $t_{i}=t_{i}^{\prime}$ for $i=q+1, \ldots, k$. Hence, for $\delta, \delta^{\prime} \in \Delta_{k}$, we obtain an order $\leq$ on the finite set $\mathscr{M}_{\delta \delta^{\prime}}$.

Each non-zero element $f$ of $I_{\delta \delta^{\prime}}$ can be written (uniquely) in the form $f=\lambda_{1} w_{1}+$ $\cdots+\lambda_{r} w_{r}$, where $w_{1}, \ldots, w_{r} \in \mathscr{M}_{\delta \delta^{\prime}}, w_{1}>\cdots>w_{r}$, and $\lambda_{1}, \ldots, \lambda_{r} \in \mathbb{F} \backslash\{0\}$. The largest monomial $w_{1}$ is called the leading monomial of $f$, and we write $w_{1}=\operatorname{lead}(f)$. 
We shall now define a quasi-order on $\mathscr{M}$. Let $\delta, \delta^{\prime} \in \Delta_{k}$ and $\varepsilon, \varepsilon^{\prime} \in \Delta_{l}$. Let $v \in \mathscr{M}_{\delta \delta^{\prime}}$ and $w \in \mathscr{M}_{\varepsilon \varepsilon^{\prime}}$, where

$$
v=\left(x_{1}^{\alpha_{1}} \cdots x_{k}^{\alpha_{k}} \otimes x_{1}^{\alpha_{1}^{\prime}} \cdots x_{k}^{\alpha_{k}^{\prime}}\right) e_{\delta \delta^{\prime}}, \quad w=\left(x_{1}^{\beta_{1}} \cdots x_{l}^{\beta_{l}} \otimes x_{1}^{\beta_{1}^{\prime}} \cdots x_{l}^{\beta_{l}^{\prime}}\right) e_{\varepsilon \varepsilon^{\prime}} .
$$

We write $v \preccurlyeq w$ if there is a one-one order-preserving map $\phi:\{1, \ldots, k\} \rightarrow$ $\{1, \ldots, l\}$ together with a homomorphism $\psi: A_{k} \rightarrow A_{l}$ with the following three properties.

(i) For $i=1, \ldots, k$, we have $x_{i} \psi=z_{i} x_{i \phi}$ for some $z_{i} \in\left\langle x_{1}, \ldots, x_{i \phi-1}\right\rangle$.

(ii) $\psi$ induces a homomorphism of $K \oplus K$-forms from $\left(\tilde{A}_{k}, \theta_{\delta \delta^{\prime}}\right)$ to $\left(\tilde{A}_{l}, \theta_{\varepsilon \varepsilon^{\prime}}\right)$.

(iii) For $i=1, \ldots, k$, we have $\alpha_{i}=\beta_{i \phi}$ and $\alpha_{i}^{\prime}=\beta_{i \phi}^{\prime}$.

It is straightforward to check that $(\mathscr{M}, \preccurlyeq)$ is a quasi-ordered set. Thus $\left(\mathscr{M}^{*}, \preccurlyeq\right)$ is quasi-ordered. Let $\mathscr{Z}$ be the set of all $N$-regular $(K \oplus K, T)$-forms as defined in

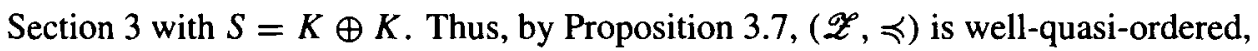
where $\preccurlyeq$ is as defined in Section 3. Let $v \in \mathscr{M}^{*}$, where

$$
v=\left(x_{1}^{\alpha_{1}} \cdots x_{k}^{\alpha_{k}} \otimes x_{1}^{\alpha_{1}^{\prime}} \cdots x_{k}^{\alpha_{k}^{\prime}}\right) e_{\delta \delta^{\prime}},
$$

with $\delta, \delta^{\prime} \in \Delta_{k}$ and $\theta_{\delta \delta^{\prime}}$ regular. Then we can define $Z(v) \in \mathscr{Z}$ by

$$
Z(v)=\left(\tilde{A}_{k}, \theta_{\delta \delta^{\prime}},\left\{\tilde{x}_{1}, \ldots, \tilde{x}_{k}\right\}, \mathbf{t}\right),
$$

where $\mathbf{t}=\left(\left(\alpha_{1}, \alpha_{1}^{\prime}\right), \ldots,\left(\alpha_{k}, \alpha_{k}^{\prime}\right)\right)$. It is straightforward to verify that if $v$ and $w$ are elements of $\mathscr{M}^{*}$ such that $Z(v) \preccurlyeq Z(w)$ then $v \preccurlyeq w$. Hence Proposition 3.7 gives the following result.

PROPOSITION 5.8. The set $\left(\mathscr{M}^{*}, \preccurlyeq\right)$ is well-quasi-ordered.

If $S$ is any set of elements of $\mathbb{F}(A \times A)$ we write $L_{\psi}(S)$ for the $\Psi$-closed left ideal generated by $S$.

LEMMA 5.9. Let $f \in I_{\delta \delta^{\prime}} \backslash\{0\}$ and $g \in I_{\varepsilon \varepsilon^{\prime}} \backslash\{0\}$ where $\delta, \delta^{\prime} \in \Delta_{k}$ and $\varepsilon, \varepsilon^{\prime} \in \Delta_{l}$. Suppose that lead $(f) \preccurlyeq \operatorname{lead}(g)$. Then there exists $g^{*} \in I_{\varepsilon \varepsilon^{\prime}}$ such that $L_{\Psi}\{f, g\}=$ $L_{\psi}\left\{f, g^{*}\right\}$ and either $g^{*}=0$ or $\operatorname{lead}\left(g^{*}\right)<\operatorname{lead}(g)$.

PROOF. Write $f=\lambda_{1} v_{1}+\cdots+\lambda_{r} v_{r}$, where $v_{i} \in \mathscr{M}_{\delta \delta^{\prime}}$ and $\lambda_{i} \in \mathbb{F} \backslash\{0\}$ for all $i$, and where $v_{i}<v_{1}$ for all $i \geq 2$. Similarly, write $g=\mu_{1} w_{1}+\cdots+\mu_{s} w_{s}$, where $w_{i} \in \mathscr{M}_{\varepsilon \varepsilon^{\prime}}$ and $\mu_{i} \in \mathbb{F} \backslash\{0\}$ for all $i$, and where $w_{i}<w_{1}$ for all $i \geq 2$. Write $v=v_{1}=\operatorname{lead}(f)$ and $w=w_{1}=\operatorname{lead}(g)$. Thus $v \preccurlyeq w$. We use the notation for $v$ and $w$ given in the definition of $\preccurlyeq$. Let $\phi$ and $\psi$ be as in that definition. Let $h_{1}$ and $h_{2}$ be the elements of $\mathbb{F}\left(A_{l} \times A_{l}\right)$ defined by

$$
h_{1}=\left(x_{1 \phi}^{\alpha_{1}} \cdots x_{k \phi}^{\alpha_{k}} \otimes x_{1 \phi}^{\alpha_{1}^{\prime}} \cdots x_{k \phi}^{\alpha_{k}^{\prime}}\right)\left(\left(x_{1}^{\alpha_{1}} \cdots x_{k}^{\alpha_{k}} \otimes x_{1}^{\alpha_{i}^{\prime}} \cdots x_{k}^{\alpha_{k}^{\prime}}\right)^{-1} \psi\right)
$$


and $h_{2}=\prod_{j \in C} x_{j}^{\beta_{j}} \otimes \prod_{j \in C} x_{j}^{\beta_{j}^{\prime}}$, where $C=\{1, \ldots, l\} \backslash\{1 \phi, \ldots, k \phi\}$. Then

$$
h_{1}(v \psi)=\left(x_{1 \phi}^{\alpha_{1}} \cdots x_{k \phi}^{\alpha_{k}} \otimes x_{1 \phi}^{\alpha_{1}^{\prime}} \cdots x_{k \phi}^{\alpha_{k}^{\prime}}\right)\left(e_{\delta \delta^{\prime}} \psi\right)
$$

and so, by Lemma 5.4 ,

$$
h_{1}(v \psi) e_{\varepsilon \varepsilon^{\prime}}=\left(x_{1 \phi}^{\alpha_{1}} \cdots x_{k \phi}^{\alpha_{k}} \otimes x_{1 \phi}^{\alpha_{1}^{\prime}} \cdots x_{k \phi}^{\alpha_{k}^{\prime}}\right) e_{\varepsilon \varepsilon^{\prime}}=\left(x_{1 \phi}^{\beta_{1 \phi}} \cdots x_{k \phi}^{\beta_{k \phi}} \otimes x_{1 \phi}^{\beta_{1 \phi}^{\prime}} \cdots x_{k \phi}^{\beta_{k \phi}^{\prime}}\right) e_{\varepsilon \varepsilon^{\prime}} .
$$

Therefore $h_{2} h_{1}(v \psi) e_{\varepsilon \varepsilon^{\prime}}=\left(x_{1}^{\beta_{1}} \cdots x_{l}^{\beta_{i}} \otimes x_{1}^{\beta_{1}^{\prime}} \cdots x_{l}^{\beta_{i}^{\prime}}\right)\left(a \otimes a^{\prime}\right) e_{\varepsilon \varepsilon^{\prime}}$, where $a, a^{\prime} \in A_{l}^{\prime}$. By (5.2), $\left(a \otimes a^{\prime}\right) e_{\varepsilon \varepsilon^{\prime}}=\lambda e_{\varepsilon \varepsilon^{\prime}}$ where $\lambda \in \mathbb{F} \backslash\{0\}$. Hence

$$
h_{2} h_{1}(v \psi) e_{\varepsilon \varepsilon^{\prime}}=\lambda\left(x_{1}^{\beta_{1}} \cdots x_{l}^{\beta_{l}} \otimes x_{1}^{\beta_{1}^{\prime}} \cdots x_{l}^{\beta_{l}^{\prime}}\right) e_{\varepsilon \varepsilon^{\prime}}=\lambda w .
$$

Now let $u$ be an element of $\mathscr{M}_{\delta \delta^{\prime}}$ such that $u<v$. Write $u=\left(x_{1}^{\gamma_{1}} \cdots x_{k}^{\gamma_{k}} \otimes x_{1}^{\gamma_{1}^{\prime}} \cdots x_{k}^{\gamma_{\gamma}^{\prime}}\right) e_{\delta \delta^{\prime}}$. Thus there exists $q \in\{1, \ldots, k\}$ such that $\left(\gamma_{q}, \gamma_{q}^{\prime}\right)<\left(\alpha_{q}, \alpha_{q}^{\prime}\right)$ but $\left(\gamma_{i}, \gamma_{i}^{\prime}\right)=\left(\alpha_{i}, \alpha_{i}^{\prime}\right)$ for $i=q+1, \ldots, k$. We can write

$$
\begin{aligned}
\left(x_{1}^{\alpha_{1}} \cdots x_{k}^{\alpha_{k}} \otimes x_{1}^{\alpha_{1}^{\prime}} \cdots x_{k}^{\alpha_{k}^{\prime}}\right)^{-1} & \left(x_{1}^{\gamma_{1}} \cdots x_{k}^{\gamma_{k}} \otimes x_{1}^{\gamma_{1}^{\prime}} \cdots x_{k}^{\gamma_{k}^{\prime}}\right) \\
= & \left(x_{1}^{\gamma_{1}-\alpha_{1}} \cdots x_{k}^{\gamma_{k}-\alpha_{k}} \otimes x_{1}^{\gamma_{1}^{\prime}-\alpha_{1}^{\prime}} \cdots x_{k}^{\gamma_{k}^{\prime}-\alpha_{k}^{\prime}}\right)\left(b \otimes b^{\prime}\right)
\end{aligned}
$$

where $b, b^{\prime} \in A_{k}^{\prime}$. By (5.2), $\left(b \otimes b^{\prime}\right) \psi e_{\varepsilon \varepsilon^{\prime}}=v e_{\varepsilon \varepsilon^{\prime}}$ where $v \in \mathbb{F} \backslash\{0\}$. Hence

$$
\begin{aligned}
h_{1}(u \psi) e_{\varepsilon \varepsilon^{\prime}} & =v\left(x_{1 \phi}^{\alpha_{1}} \cdots x_{k \phi}^{\alpha_{k}} \otimes x_{1 \phi}^{\alpha_{1}^{\prime}} \cdots x_{k \phi}^{\alpha_{k}^{\prime}}\right)\left(\left(x_{1}^{\gamma_{1}-\alpha_{1}} \cdots x_{k}^{\gamma_{k}-\alpha_{k}} \otimes x_{1}^{\gamma_{1}^{\prime}-\alpha_{1}^{\prime}} \cdots x_{k}^{\gamma_{k}^{\prime}-\alpha_{k}^{\prime}}\right) \psi\right) e_{\varepsilon \varepsilon^{\prime}} \\
& =v\left(x_{1 \phi}^{\alpha_{1}} \cdots x_{k \phi}^{\alpha_{k}} \otimes x_{1 \phi}^{\alpha_{1}^{\prime}} \cdots x_{k \phi}^{\alpha_{k}^{\prime}}\right)\left(\left(x_{1}^{\gamma_{1}-\alpha_{1}} \cdots x_{q}^{\gamma_{q}-\alpha_{q}} \otimes x_{1}^{\gamma_{1}^{\prime}-\alpha_{1}^{\prime}} \cdots x_{q}^{\gamma_{q}^{\prime}-\alpha_{q}^{\prime}}\right) \psi\right) e_{\varepsilon \varepsilon^{\prime}} .
\end{aligned}
$$

From the properties of $\psi$ we calculate that

$$
\begin{aligned}
\left(\left(x_{1}^{\gamma_{1}-\alpha_{1}} \cdots x_{q}^{\gamma_{q}-\alpha_{q}}\right.\right. & \left.\left.\otimes x_{1}^{\gamma_{1}^{\prime}-\alpha_{1}^{\prime}} \cdots x_{q}^{\gamma_{q}^{\prime}-\alpha_{q}^{\prime}}\right) \psi\right) e_{\varepsilon \varepsilon^{\prime}} \\
& =v^{\prime}\left(x_{1}^{\rho_{1}} \cdots x_{q \phi-1}^{\rho_{q \phi-1}} x_{q \phi}^{\gamma_{q}-\alpha_{q}} \otimes x_{1}^{\rho_{1}^{\prime}} \cdots x_{q \phi-1}^{\rho_{q \phi-1}^{\prime}} x_{q \phi}^{\gamma_{q}^{\prime}-\alpha_{q}^{\prime}}\right) e_{\varepsilon \varepsilon^{\prime}}
\end{aligned}
$$

where $v^{\prime} \in \mathbb{F} \backslash\{0\}$ and $\rho_{1}, \ldots, \rho_{q \phi-1}, \rho_{1}^{\prime}, \ldots, \rho_{q \phi-1}^{\prime} \in \mathbb{Z} / n \mathbb{Z}$. Hence $h_{1}(u \psi) e_{\varepsilon \varepsilon^{\prime}}$ has the form

$$
v^{\prime \prime}\left(x_{1}^{\sigma_{1}} \cdots x_{q \phi-1}^{\sigma_{q \phi-1}} x_{q \phi}^{\gamma_{q}} x_{(q+1) \phi}^{\alpha_{q+1}} \cdots x_{k \phi}^{\alpha_{k}} \otimes x_{1}^{\sigma_{1}^{\prime}} \cdots x_{q \phi-1}^{\sigma_{q \phi-1}^{\prime}} x_{q \phi}^{\gamma_{q}^{\prime}} x_{(q+1) \phi}^{\alpha_{q+1}^{\prime}} \cdots x_{k \phi}^{\alpha_{k}^{\prime}}\right) e_{\varepsilon \varepsilon^{\prime}}
$$

where $v^{\prime \prime} \in \mathbb{F} \backslash\{0\}$ and $\sigma_{1}, \ldots, \sigma_{q \phi-1}, \sigma_{1}^{\prime}, \ldots, \sigma_{q \phi-1}^{\prime} \in \mathbb{Z} / n \mathbb{Z}$. Therefore $h_{2} h_{1}(u \psi) e_{\varepsilon \varepsilon^{\prime}}$ is a non-zero scalar multiple of a monomial of the form

$$
\left(x_{1}^{\tau_{1}} \cdots x_{q \phi-1}^{\tau_{q \phi-1}} x_{q \phi}^{\gamma_{q}} x_{q \phi+1}^{\beta_{q \phi+1}} \cdots x_{l}^{\beta_{i}} \otimes x_{1}^{\tau_{1}^{\prime}} \cdots x_{q \phi-1}^{\tau_{q \phi-1}^{\prime}} x_{q \phi}^{\gamma_{q}^{\prime}} x_{q \phi+1}^{\beta_{q \phi+1}^{\prime}} \cdots x_{l}^{\beta_{i}^{\prime}}\right) e_{\varepsilon \varepsilon^{\prime}}
$$

where $\tau_{1}, \ldots, \tau_{q \phi-1}, \tau_{1}^{\prime}, \ldots, \tau_{q \phi-1}^{\prime} \in \mathbb{Z} / n \mathbb{Z}$. Since $\left(\gamma_{q}, \gamma_{q}^{\prime}\right)<\left(\alpha_{q}, \alpha_{q}^{\prime}\right)=\left(\beta_{q \phi}, \beta_{q \phi}^{\prime}\right)$, this monomial is smaller than $w$.

Since $h_{2} h_{1}(f \psi) e_{\varepsilon \varepsilon^{\prime}}=\lambda_{1} h_{2} h_{1}\left(v_{1} \psi\right) e_{\varepsilon \varepsilon^{\prime}}+\cdots+\lambda_{r} h_{2} h_{1}\left(v_{r} \psi\right) e_{\varepsilon \varepsilon^{\prime}}$, we see that $h_{2} h_{1}(f \psi) e_{\varepsilon \varepsilon^{\prime}}$ has leading monomial $w$ with coefficient $\lambda_{1} \lambda$. Also, since $\psi$ extends to an element of $\Psi$, we have $h_{2} h_{1}(f \psi) e_{\varepsilon \varepsilon^{\prime}} \in L_{\psi}\{f\}$. Let $g^{*}=g-\mu_{1} \lambda_{1}^{-1} \lambda^{-1} h_{2} h_{1}(f \psi) e_{\varepsilon \varepsilon^{\prime}}$. Then $g^{*}$ has the required properties. 
Now we are in a position to complete the proof of Theorem C. Let $J$ be a $\Psi$-closed left ideal of $\mathbb{F}(A \times A)$. It suffices to prove that $J$ is finitely generated as a $\Psi$-closed left ideal. By Proposition 5.8, there exists a finite set $S$ of regular elements of $J$ such that for every regular element $g$ of $J$ there exists $f \in S$ such that lead $(f) \preccurlyeq \operatorname{lead}(g)$. We claim that $J=L_{\psi}(S)$. By Lemma 5.7, it suffices to show that every regular element of $J$ belongs to $L_{\Psi}(S)$. Suppose, in order to get a contradiction, that this is not so, and let $g$ be a regular element of $J$ such that $g \notin L_{\Psi}(S)$. Suppose $g \in I_{\varepsilon \varepsilon^{\prime}}$. Choose $g$ with the given properties such that lead $(g)$ is as small as possible in the finite set $\left(\mathscr{M}_{\varepsilon \varepsilon^{\prime}}, \leq\right)$. There exists $f \in S$ such that lead $(f) \preccurlyeq \operatorname{lead}(g)$. By Lemma 5.9, there exists $g^{*} \in I_{\varepsilon \varepsilon^{\prime}}$ such that $L_{\psi}\{f, g\}=L_{\psi}\left\{f, g^{*}\right\}$ and either $g^{*}=0$ or lead $\left(g^{*}\right)<\operatorname{lead}(g)$. Since $g \notin L_{\Psi}\{f\}$, we have $g^{*} \neq 0$. Since $g^{*} \in L_{\Psi}\{f, g\} \subseteq J$, the choice of $g$ gives that $g^{*} \in L_{\Psi}(S)$. Hence $g \in L_{\psi}\left\{f, g^{*}\right\} \subseteq L_{\psi}(S)$ and we have the required contradiction.

\section{Proof of Theorem D}

Let $n, A, \Psi, \mathbb{F}$ and $R$ be as in Section 1 , where $\mathbb{F}$ is a field of characteristic not dividing $n$. Let $\overline{\mathbb{F}}$ be the algebraic closure of $\mathbb{F}$. The subalgebra $\overline{\mathbb{F}} \otimes_{\mathbb{F}} R$ of $\overline{\mathbb{F}}(A \times A)$ corresponds to $R$ in $\mathbb{F}(A \times A)$. If $M$ is an $(R, \Psi)$-submodule of $\mathbb{F}(A \times A)$ which contains $R$, then $\overline{\mathbb{F}} \otimes_{\mathbb{F}} M$ is an $\left(\overline{\mathbb{F}} \otimes_{\mathbb{F}} R, \Psi\right)$-submodule of $\overline{\mathbb{F}}(A \times A)$ which contains $\overline{\mathbb{F}} \otimes_{\mathbb{F}} R$, and $M=\mathbb{F}(A \times A) \cap \overline{\mathbb{F}} \otimes_{\mathbb{F}} M$. Therefore, to prove Theorem $\mathrm{D}$, we may assume that $\overline{\mathbb{F}}=\mathbb{F}$.

We shall use the notation of Section 5. If $n \leq 2$, then Theorem $\mathrm{D}$ follows from Proposition 4.5. Thus, as in Section 5, we assume that $n>2$, so that $n_{0}>1$.

Let $P$ be the subgroup of $A \times A$ defined by $P=\left\{\left(c, c^{-1}\right): c \in A^{\prime}\right\}$ and let $H=(A \times A) / P$. Note that $P$ is a $\Psi$-closed subgroup of $A \times A$, so each element of $\Psi$ induces endomorphisms of $H$ and $\mathbb{F} H$. For $i, j \in \mathbb{N}$, let $c_{i j}$ be the element of $H$ given by $c_{i j}=\left(\left[x_{i}, x_{j}\right], 1\right) P=\left(1,\left[x_{i}, x_{j}\right]\right) P$.

For each positive integer $k$, let $H_{k}$ be the subgroup of $H$ generated by the elements $\left(x_{i}, 1\right) P$ and $\left(1, x_{i}\right) P$ for $i=1, \ldots, k$. It is easily verified that $H_{k}^{\prime}$ is a free abelian group of exponent $n_{0}$ with basis $\left\{c_{i j}: 1 \leq i<j \leq k\right\}$. Furthermore, there are isomorphisms from $A_{k}^{\prime}$ to $H_{k}^{\prime}$ and from $\mathbb{F} A_{k}^{\prime}$ to $\mathbb{F} H_{k}^{\prime}$ given by $\left[x_{i}, x_{j}\right] \mapsto c_{i j}$ for all $i, j$. If $\psi: A_{k} \rightarrow A_{l}$ is a homomorphism, where $k, l \in \mathbb{N}$, then the associated homomorphism $\psi: A_{k} \times A_{k} \rightarrow A_{l} \times A_{l}$ yields homomorphisms $\bar{\psi}: H_{k} \rightarrow H_{l}$ and $\bar{\psi}: \mathbb{F} H_{k} \rightarrow \mathbb{F} H_{l}$.

Let $K=\mathbb{Z} / n_{0} \mathbb{Z}$, and let $Q_{k}$ and $\Delta_{k}$ be as in Section 5. For each $\delta \in \Delta_{k}$, let $\chi_{\delta}$ and $e_{\delta}$ be defined as in Section 5, but with respect to $H_{k}^{\prime}$ rather than $A_{k}^{\prime}$. Thus $\chi_{\delta}$ is a character of $H_{k}^{\prime}$ and $e_{\delta}$ is an idempotent of $\mathbb{F} H_{k}^{\prime}$. Results (5.2)-(5.5) apply just as before. For $\delta \in \Delta_{k}$ we define $J_{\delta}=\left(\mathbb{F} H_{k}\right) e_{\delta}$. Thus $J_{\delta}$ is the ideal of $\mathbb{F} H_{k}$ generated by $e_{\delta}$, and we have $\mathbb{F} H_{k}=\bigoplus_{\delta \in \Delta_{k}} J_{\delta}$. 
For each $k$ we write $Q_{k}^{0}=Q_{k} \backslash\{(1,2),(3,4), \ldots\}$. An element $\delta$ of $\Delta_{k}$ will be cafled standard if $\delta(i, j)=0$ (equivalently, $\chi_{\delta}\left(c_{i j}\right)=1$ ) for all $(i, j) \in Q_{k}^{0}$. We write $\Delta_{k}^{*}$ for the set of all standard elements of $\Delta_{k}$ and $\Delta_{k}^{0}=\Delta_{k} \backslash \Delta_{k}^{*}$.

For each $\delta \in \Delta_{k}$, let $\theta_{\delta}$ be the alternating $K$-form on $\tilde{A}_{k}$ defined as in Section 5 . Thus $\left(\tilde{A}_{k}, \theta_{\delta}\right)$ is standard with respect to $\left\{\tilde{x}_{1}, \ldots, \tilde{x}_{k}\right\}$ (in the terminology of Section 3 ) if and only if $\delta$ is standard, that is, $\delta \in \Delta_{k}^{*}$.

LEMMA 6.1. Let $\delta \in \Delta_{k}$. Then there exists $\eta \in \operatorname{Aut}\left(A_{k}\right)$ such that, for the induced automorphism $\bar{\eta}: \mathbb{F} H_{k} \rightarrow \mathbb{F} H_{k}$, we have $e_{\delta} \bar{\eta}=e_{\varepsilon}$ where $\varepsilon \in \Delta_{k}^{*}$.

ProOF. By Lemma 3.8 there is a basis $\left\{\tilde{a}_{1}, \ldots, \tilde{a}_{k}\right\}$ of $\tilde{A}_{k}$ such that $\left(\tilde{A}_{k}, \theta_{\delta}\right)$ is standard with respect to this basis. As in the proof of Lemma 5.5, there is a free generating set $\left\{y_{1}, \ldots, y_{k}\right\}$ of $A_{k}$ such that $\tilde{y}_{i}=\tilde{a}_{i}$ for $i=1, \ldots, k$. Let $\eta$ be the automorphism of $A_{k}$ satisfying $y_{i} \eta=x_{i}$ for $i=1, \ldots, k$. Note that $\eta$ acts on $A_{k}^{\prime}$ just as $\bar{\eta}$ acts on $H_{k}^{\prime}$. Thus Lemma 5.1 shows that $e_{\delta} \bar{\eta}=e_{\varepsilon}$, where $\varepsilon \in \Delta_{k}$ and $\theta_{\varepsilon}\left(\tilde{x}_{i}, \tilde{x}_{j}\right)=\theta_{\delta}\left(\tilde{y}_{i}, \tilde{y}_{j}\right)$ for all $i, j$. Thus $\left(\tilde{A}_{k}, \theta_{\varepsilon}\right)$ is standard with respect to $\left\{\tilde{x}_{1}, \ldots, \bar{x}_{k}\right\}$, that is, $\varepsilon \in \Delta_{k}^{*}$.

Since $\mathbb{F} H^{\prime}$ is a subalgebra of $\mathbb{F} H$, we may regard $\mathbb{F} H$ as a left $\mathbb{F} H^{\prime}$-module. Following the terminology of Section 1, we shall consider $\left(\mathbb{F} H^{\prime}, \Psi\right)$-submodules of $\mathbb{F} H$. A non-zero element $w$ of $\mathbb{F} H$ will be called standard if $w \in J_{\delta}$ for some $k$ and some $\delta \in \Delta_{k}^{*}$.

LEMMA 6.2. Every $\left(\mathbb{F} H^{\prime}, \Psi\right)$-submodule of $\mathbb{F} H$ is generated, as a $\Psi$-closed vector space, by standard elements.

PROOF. This is similar to the proof of Lemma 5.7, with Lemma 6.1 taking the place of Lemma 5.5.

Let $C$ be the subgroup of $H$ generated by all elements $c_{i j}$ for which $i<j$ and $(i, j) \notin\{(1,2),(3,4), \ldots\}$. Let $\rho$ be the natural homomorphism $\rho: H \rightarrow H / C$. We also denote by $\rho$ the associated homomorphisms $H_{k} \rightarrow H / C$ and $\mathbb{F} H_{k} \rightarrow \mathbb{F}(H / C)$. Clearly the kernel of $\rho: H_{k} \rightarrow H / C$ is the subgroup of $H_{k}$ generated by all $c_{i j}$ for which $(i, j) \in Q_{k}^{0}$. Thus the kernel of $\rho: \mathbb{F} H_{k} \rightarrow \mathbb{F}(H / C)$ is the ideal generated by the elements $c_{i j}-1$ for $(i, j) \in Q_{k}^{0}$. We write $\left(\mathbb{F} H_{k}\right)^{*}=\bigoplus_{\delta \in \Delta_{k}^{*}} J_{\delta}$ and $\left(\mathbb{F} H_{k}\right)^{0}=\bigoplus_{\delta \in \Delta_{k}^{0}} J_{\delta}$.

LEMMA 6.3. The kernel of $\rho: \mathbb{F} H_{k} \rightarrow \mathbb{F}(H / C)$ is $\left(\mathbb{F} H_{k}\right)^{0}$.

Proof. Let $\delta \in \Delta_{k}^{0}$. Then $\chi_{\delta}\left(c_{i j}\right) \neq 1$ for some $(i, j) \in Q_{k}^{0}$. By (5.2), $\left(c_{i j}-1\right) e_{\delta}$ is a non-zero scalar multiple of $e_{\delta}$. But clearly $\left(c_{i j}-1\right) e_{\delta} \in \operatorname{ker}(\rho)$. Thus $e_{\delta} \in \operatorname{ker}(\rho)$. It follows that $J_{\delta} \subseteq \operatorname{ker}(\rho)$ and so $\left(\mathbb{F} H_{k}\right)^{0} \subseteq \operatorname{ker}(\rho)$. 
Let $(i, j) \in Q_{k}^{0}$. Then, for $\varepsilon \in \Delta_{k}^{*}$, we have $\left(c_{i j}-1\right) e_{\varepsilon}=\left(\chi_{\varepsilon}\left(c_{i j}\right)-1\right) e_{\varepsilon}=0$. Hence $c_{i j}-1=\left(c_{i j}-1\right) \sum_{\delta \in \Delta_{k}} e_{\delta}=\left(c_{i j}-1\right) \sum_{\delta \in \Delta_{k}^{0}} e_{\delta}$. Hence $c_{i j}-1$ belongs to the ideal $\left(\mathbb{F} H_{k}\right)^{0}$. Since this holds for all $(i, j) \in Q_{k}^{0}$ we obtain $\operatorname{ker}(\rho) \subseteq\left(\mathbb{F} H_{k}\right)^{0}$.

For $k \in \mathbb{N}$, let $\psi_{k}$ be the endomorphism of $A$ defined by $x_{i} \psi_{k}=1$ for $i>k$ and $x_{i} \psi_{k}=x_{i}$ for $i \leq k$. Also write $\psi_{k}$ for the induced endomorphisms of $H$ and $\mathbb{F} H$.

LEMMA 6.4. Let $u \in\left(\mathbb{F} H_{k}\right)^{*}$ and let $l \geq k$. Then there exists $v \in\left(\mathbb{F} H_{l}\right)^{*}$ such that $v \psi_{k}=u$ and $v \rho=u \rho$.

Proof. Let $B$ be the subgroup of $H_{l}^{\prime}$ generated by all elements $c_{i j}$ for $(i, j) \in$ $Q_{l}^{0} \backslash Q_{k}^{0}$. Let $v=u\left(|B|^{-1} \sum_{h \in B} h\right)$. Clearly $v \psi_{k}=u$ and $v \rho=u \rho$. To prove that $v \in\left(\mathbb{F} H_{l}\right)^{*}$ it is enough to show that $v e_{\varepsilon}=0$ for all $\varepsilon \in \Delta_{l}^{0}$.

Let $\varepsilon \in \Delta_{l}^{0}$. Then there exists $(i, j) \in Q_{l}^{0}$ such that $\chi_{\varepsilon}\left(c_{i j}\right) \neq 1$. We consider two cases. Suppose first that $(i, j) \in Q_{k}^{0}$. Then the restriction of $\chi_{\varepsilon}$ to $H_{k}^{\prime}$ has the form $\chi_{\delta^{\prime}}$ for some $\delta^{\prime} \in \Delta_{k}^{0}$. Then for all $\delta \in \Delta_{k}^{*}$ we have $e_{\delta} e_{\varepsilon}=\chi_{\delta^{\prime}}\left(e_{\delta}\right) e_{\varepsilon}=0$, by (5.2) and (5.4). Hence $u e_{\varepsilon}=0$ and so $v e_{\varepsilon}=0$. Suppose secondly that $(i, j) \in Q_{l}^{0} \backslash Q_{k}^{0}$. Then $\sum_{h \in B} h$ can be written as $w\left(1+c_{i j}+\cdots+c_{i j}^{n_{0}}\right)$ for some $w \in \mathbb{F} H_{l}^{\prime}$. Since $\chi_{\varepsilon}\left(c_{i j}\right)$ is a non-trivial $n_{0}$-th root of unity, $\chi_{\varepsilon}\left(1+c_{i j}+\cdots+c_{i j}^{n_{0}}\right)=0$. Thus $\left(1+c_{i j}+\cdots+c_{i j}^{n_{0}}\right) e_{\varepsilon}=0$ and so $v e_{\varepsilon}=0$.

LEMMA 6.5. Suppose that $M_{1}$ and $M_{2}$ are $\left(\mathbb{F} H^{\prime}, \Psi\right)$-submodules of $\mathbb{F} H$ such that $M_{1} \rho=M_{2} \rho$. Then $M_{1}=M_{2}$.

Proof. Suppose, in order to get a contradiction, that $M_{1} \neq M_{2}$. Without loss of generality we may assume that $M_{1} \nsubseteq M_{2}$. By Lemma 6.2 there exist $k$ and $\delta \in \Delta_{k}^{*}$ such that $M_{1} \cap J_{\delta} \nsubseteq M_{2}$. Hence there exists $u \in\left(\mathbb{F} H_{k}\right)^{*}$ such that $u \in M_{1} \backslash M_{2}$. By hypothesis there exists $w \in M_{2}$ such that $u \rho=w \rho$. Choose $l \geq k$ such that $w \in \mathbb{F} H_{l}$. Then $w=w^{*}+w^{0}$ where $w^{*} \in\left(\mathbb{F} H_{l}\right)^{*}$ and $w^{0} \in\left(\mathbb{F} H_{l}\right)^{0}$. Since $M_{2}$ is an $\mathbb{F} H^{\prime}$-module, $w^{*} \in M_{2}$. Also $u \rho=w \rho=w^{*} \rho$. By Lemma 6.4 there exists $v \in\left(\mathbb{F} H_{l}\right)^{*}$ such that $v \psi_{k}=u$ and $v \rho=u \rho$. Thus $v \rho=w^{*} \rho$. By Lemma 6.3, this gives $v=w^{*} \in M_{2}$. Hence $u=v \psi_{k} \in M_{2}$, which is a contradiction.

Now we return to the group $H / C$. Recall that $H=(A \times A) / P$. For each $i \in \mathbb{N}$, let $G_{i}$ be the subgroup of $H / C$ generated by the four elements $\left(\left(x_{2 i-1}, 1\right) P\right) \rho$, $\left(\left(1, x_{2 i-1}\right) P\right) \rho,\left(\left(x_{2 i}, 1\right) P\right) \rho$ and $\left(\left(1, x_{2 i}\right) P\right) \rho$. Write $G=G_{1}$. Thus $G$ is a finite group. It is easily verified that $H / C$ is the direct product of the groups $G_{i}$, and, for each $i$, there is an obvious isomorphism from $G$ to $G_{i}$. Thus we may identify $H / C$ with the direct power $D$ of $G$ considered in Section 4 . Let $\Xi$ be the set of endomorphisms of $D$ defined in Section 4. 
LEMMA 6.6. Let $M$ be a $\Psi$-closed subspace of $\mathbb{F} H$. Then $M \rho$ is a $\Xi$-closed subspace of $\mathbb{F} D$.

PROOF. Let $\xi \in \Xi$ and suppose that $\xi$ is associated with $\phi, X$ and $\sigma$ in the notation of Section 4. It suffices to show that there exists an endomorphism $\psi$ of $A$ such that the induced endomorphism of $H$ leaves $C$ invariant and induces $\xi$ on $H / C$. To simplify the notation we rewrite the generators of $A$ by setting $y_{i}=x_{2 i-1}$ and $z_{i}=x_{2 i}$ for all $i \in \mathbb{N}$. We define a homomorphism $\psi: A \rightarrow A$ by

$$
y_{i} \psi=y_{i \phi} \prod_{\substack{j \in X \\ j \sigma=i \phi}} y_{j}, \quad z_{i} \psi=z_{i \phi} \prod_{\substack{j \in X \\ j \sigma=i \phi}} z_{j},
$$

for each $i$. The products are taken over all those values of $j$, if any, which lie in $X$ and satisfy $j \sigma=i \phi$, and the terms $y_{j}$ and $z_{j}$ are taken in increasing order of $j$ (this is an arbitrary choice). It is straightforward to verify that $\psi$ has the required properties.

By Proposition 4.4 together with Lemma 6.5 and Lemma 6.6 we obtain

LEMMA 6.7. The maximal condition holds for $\left(\mathbb{F} H^{\prime}, \Psi\right)$-submodules of $\mathbb{F} H$.

Consider the natural homomorphism $\pi: A \times A \rightarrow H$ with kernel $P$, and let $I$ be the kernel of the corresponding homomorphism $\pi: \mathbb{F}(A \times A) \rightarrow \mathbb{F} H$.

LEMMA 6.8. The maximal condition holds for $(R, \Psi)$-submodules of $\mathbb{F}(A \times A)$ which contain $I$.

ProOF. By Lemma 6.7 it suffices to show that if $M$ is an $(R, \Psi)$-submodule of $\mathbb{F}(A \times A)$ which contains $I$ then $M \pi$ is an $\left(\mathbb{F} H^{\prime}, \Psi\right)$-submodule of $\mathbb{F} H$. It is clear that $M \pi$ is $\Psi$-closed, by definition of the action of $\Psi$ on $\mathbb{F} H$. Also, $M \pi$ is a left $R \pi$ submodule of $\mathbb{F} H$. Thus it suffices to show that $H^{\prime} \subseteq R \pi$. Since $R \pi$ is an algebra, it suffices to show that $c_{i j} \in R \pi$ for all $i, j$. Note that $\left(\left[x_{i}, x_{j}\right] \otimes\left[x_{i}, x_{j}\right]\right) \pi=c_{i j}^{2}$ and $\left(\left[x_{i}, x_{j}\right] \otimes 1+1 \otimes\left[x_{i}, x_{j}\right]\right) \pi=2 c_{i j}$. Hence $c_{i j}^{2} \in R \pi$ and $2 c_{i j} \in R \pi$. If $n_{0}$ is odd then $c_{i j}^{2} \in R \pi$ gives $c_{i j} \in R \pi$. But if $n_{0}$ is even then $\mathbb{F}$ does not have characteristic 2 and $2 c_{i j} \in R \pi$ gives $c_{i j} \in R \pi$.

In the notation of Section 5 , we can write $\mathbb{F}\left(A_{k} \times A_{k}\right)=\bigoplus_{\delta, \delta^{\prime} \in \Delta_{k}} I_{\delta \delta^{\prime}}$. Note that

$$
\left(e_{\delta} \otimes 1\right) \pi=\left(1 \otimes e_{\delta}\right) \pi=e_{\delta} \in \mathbb{F} H_{k}^{\prime} .
$$

Hence, for $\delta \neq \delta^{\prime}$, we have $e_{\delta \delta^{\prime}} \pi=e_{\delta} e_{\delta^{\prime}}=0$ and so $I_{\delta \delta^{\prime}} \subseteq \operatorname{ker}(\pi)=I$. It is easily checked that $\bigoplus_{\delta \in \Delta_{k}} I_{\delta \delta}$ and $\mathbb{F} H_{k}$ have the same dimension. Hence

$$
I \cap \mathbb{F}\left(A_{k} \times A_{k}\right)=\bigoplus_{\substack{\delta, \delta^{\prime} \in \Delta_{k} \\ \delta \neq \delta^{\prime}}} I_{\delta \delta^{\prime}}
$$


LEMMA 6.9. Let $M$ be an $(R, \Psi)$-submodule of $\mathbb{F}(A \times A)$ such that $R \cap I \subseteq M \subseteq I$, and let $T$ be the largest $\Psi$-closed left ideal of $\mathbb{F}(A \times A)$ contained in $M$. Then $M=T+(R \cap I)$.

Proof. Let $L$ be the subspace of $M$ spanned by all elements of $M$ which have the form $w e_{\delta \delta^{\prime}}$ where $w \in \mathbb{F}(A \times A)$ and $\delta, \delta^{\prime} \in \Delta_{k}$ for some $k$, with $\delta \neq \delta^{\prime}$. Let $w e_{\delta \delta^{\prime}}$ be such an element of $M$. Let $\psi \in \Psi$ and $a, a^{\prime} \in A$. Choose $l \geq k$ so that $e_{\delta} \psi, e_{\delta^{\prime}} \psi \in \mathbb{F} A_{l}^{\prime}$. Since $e_{\delta} \psi$ and $e_{\delta^{\prime}} \psi$ are idempotents, we can write $e_{\delta} \psi=\sum_{\lambda \in \Lambda} e_{\lambda}$ and $e_{\delta^{\prime}} \psi=\sum_{\lambda^{\prime} \in \Lambda^{\prime}} e_{\lambda^{\prime}}$ where $\Lambda, \Lambda^{\prime} \subseteq \Delta_{l}$. But $\left(e_{\delta} \psi\right)\left(e_{\delta^{\prime}} \psi\right)=\left(e_{\delta} e_{\delta^{\prime}}\right) \psi=0$. Thus $\Lambda$ and $\Lambda^{\prime}$ are disjoint. For $\varepsilon \in \Lambda$ and $\varepsilon^{\prime} \in \Lambda^{\prime}$,

$$
\left(\left(a \otimes a^{\prime}\right) e_{\varepsilon \varepsilon^{\prime}}+\left(a^{\prime} \otimes a\right) e_{\varepsilon^{\prime} \varepsilon}\right)(w \psi)\left(e_{\delta \delta^{\prime}} \psi\right) \in M,
$$

because $M$ is an $(R, \Psi)$-module. However, $e_{\delta \delta^{\prime}} \psi=\sum_{\lambda, \lambda^{\prime}} e_{\lambda \lambda^{\prime}}$. Hence $e_{\varepsilon^{\prime} \varepsilon}\left(e_{\delta \delta^{\prime}} \psi\right)=0$ and $e_{\varepsilon \varepsilon^{\prime}}\left(e_{\delta \delta^{\prime}} \psi\right)=e_{\varepsilon \varepsilon^{\prime}}$. Therefore $\left(a \otimes a^{\prime}\right)(w \psi) e_{\varepsilon \varepsilon^{\prime}} \in M$, and so $\left(a \otimes a^{\prime}\right)(w \psi) e_{\varepsilon \varepsilon^{\prime}} \in L$. Since this holds for all $\varepsilon, \varepsilon^{\prime}$, we have $\left(a \otimes a^{\prime}\right)(w \psi)\left(e_{\delta \delta^{\prime}} \psi\right) \in L$. Therefore $L$ is a $\Psi$-closed left ideal of $\mathbb{F}(A \times A)$. We next prove that $M=L+(R \cap I)$, which will give the required result.

Let $u \in M$ and choose $k$ so that $u \in \mathbb{F}\left(A_{k} \times A_{k}\right)$. Since $M \subseteq I$ we can use (6.1) to write $u=\sum w_{\delta \delta^{\prime}} e_{\delta \delta^{\prime}}$, where the sum is over all $\delta, \delta^{\prime} \in \Delta_{k}$ with $\delta \neq \delta^{\prime}$ and each $w_{\delta \delta^{\prime}}$ belongs to $\mathbb{F}\left(A_{k} \times A_{k}\right)$. Let $\delta, \delta^{\prime} \in \Delta_{k}$ with $\delta \neq \delta^{\prime}$. Since $M$ is an $R$-module,

$$
\left(e_{\delta \delta^{\prime}}+e_{\delta^{\prime} \delta}\right) u=w_{\delta \delta^{\prime}} e_{\delta \delta^{\prime}}+w_{\delta^{\prime} \delta} e_{\delta^{\prime} \delta} \in M .
$$

Write $v=w_{\delta \delta^{\prime}}$ and $v^{\prime}=w_{\delta^{\prime} \delta}$. Then it suffices to show that $v e_{\delta \delta^{\prime}}+v^{\prime} e_{\delta^{\prime} \delta} \in L+(R \cap I)$.

Let $\tau$ be the involutory automorphism of $\mathbb{F}(A \times A)$ satisfying $\left(a \otimes a^{\prime}\right) \tau=a^{\prime} \otimes a$ for all $a, a^{\prime} \in A$. Then $w+w \tau \in R$ for all $w \in \mathbb{F}(A \times A)$. We can write

$$
v e_{\delta \delta^{\prime}}+v^{\prime} e_{\delta^{\prime} \delta}=\left(v-v^{\prime} \tau\right) e_{\delta \delta^{\prime}}+v^{\prime} e_{\delta^{\prime} \delta}+\left(v^{\prime} \tau\right) e_{\delta \delta^{\prime}} .
$$

Here

$$
v^{\prime} e_{\delta^{\prime} \delta}+\left(v^{\prime} \tau\right) e_{\delta \delta^{\prime}}=v^{\prime} e_{\delta^{\prime} \delta}+\left(v^{\prime} e_{\delta^{\prime} \delta}\right) \tau \in R \cap I \text {. }
$$

Since $R \cap I \subseteq M,(6.2)$ gives $\left(v-v^{\prime} \tau\right) e_{\delta \delta^{\prime}} \in M$, and so $\left(v-v^{\prime} \tau\right) e_{\delta \delta^{\prime}} \in L$. Therefore, by (6.2), $v e_{\delta \delta^{\prime}}+v^{\prime} e_{\delta^{\prime} \delta} \in L+(R \cap I)$, as required.

To complete the proof of Theorem $\mathrm{D}$, let $M_{1} \subseteq M_{2} \subseteq \ldots$ be an ascending chain of $(R, \Psi)$-submodules of $\mathbb{F}(A \times A)$ which contain $R$. By Lemma 6.8 the chain $M_{1}+I \subseteq M_{2}+I \subseteq \cdots$ becomes stationary. Thus it suffices to show that the chain $M_{1} \cap I \subseteq M_{2} \cap I \subseteq \cdots$ becomes stationary. For each $i$, let $T_{i}$ be the largest $\Psi$-closed left ideal of $\mathbb{F}(A \times A)$ contained in $M_{i} \cap I$. By Lemma 6.9 it suffices to show that the chain $T_{1} \subseteq T_{2} \subseteq \cdots$ becomes stationary. But this holds by Theorem $\mathrm{C}$. 


\section{References}

[1] M. D. Atkinson, 'Alternating trilinear forms and groups of exponent 6', J. Austral. Math. Soc. 16 (1973), 111-128.

[2] J. M. Brady, R. A. Bryce and J. Cossey, 'On certain abelian-by-nilpotent varieties', Bull. Austral. Math. Soc. 1 (1969), 403-416.

[3] R. M. Bryant and M. F. Newman, 'Some finitely based varieties of groups', Proc. London Math. Soc. (3) 28 (1974), 237-252.

[4] D. E. Cohen, 'On the laws of a metabelian variety', J. Algebra 5 (1967), 267-273.

[5] C. K. Gupta and A. N. Krasil'nikov, 'Some non-finitely based varieties of groups and group representations', Internat. J. Algebra Comput. 5 (1995), 343-365.

[6] G. Higman, 'Ordering by divisibility in abstract algebras', Proc. London Math. Soc. (3) 2 (1952), 326-336.

[7] —_, 'Some remarks on varieties of groups', Quart. J. Math. Oxford (2) 10 (1959), 165-178.

[8] —_, 'The orders of relatively free groups', in: Proc. Internat. Conf. Theory of Groups, Austral. Nat. Univ., 1965 (eds. L. G. Kovács and B. H. Neumann) (Gordon and Breach, New York, 1967) pp. 153-165.

[9] L. G. Kovács and M. F. Newman, 'Hanna Neumann's problems on varieties of groups', in: Proc. Second Internat. Conf. Theory of Groups, Austral. Nat. Univ., 1973 (ed. M. F. Newman), Lecture Notes in Math. 372 (Springer, Berlin, 1974) pp. 417-431.

[10] A. N. Krasil'nikov, 'The identities of a group with nilpotent commutator subgroup are finitely based', Izv. Akad. Nauk SSSR Ser. Mat. 54 (1990), 1181-1195; English translation: Math. USSR Izvestiya 37 (1991), 539-553.

[11] R. C. Lyndon, 'Two notes on nilpotent groups', Proc. Amer. Math. Soc. 3 (1952), 579-583.

[12] S. McKay, 'On centre-by-metabelian varieties of groups', Proc. London Math. Soc. (3) 24 (1972), 243-256.

[13] H. Neumann, Varieties of groups (Springer, Berlin, 1967).

[14] A. L. Shmel'kin, 'Wreath products and varieties of groups', Izv. Akad. Nauk SSSR Ser. Mat. 29 (1965), 149-170.

UMIST

PO Box 88

Manchester M60 1QD

UK

e-mail: bryant@umist.ac.uk
Moscow Pedagogical State University

14 Krasnoprudnaya ul.

Moscow 107140

Russia

Current address:

University of Brasilia

70910-900 Brasilia-DF

Brazil

e-mail: alexei@mat.unb.br 